\title{
PREDICTION OF ARTICULAR CARTILAGE REMODELING DURING DYNAMIC COMPRESSION WITH A FINITE ELEMENT MODEL
}

\author{
A Thesis \\ Presented to \\ the Faculty of California Polytechnic State University \\ San Luis Obispo
}

In Partial Fulfillment of the Requirements for the Degree

Master of Science in Mechanical Engineering

by

Kevin Yamauchi

June 2012 
(C) 2012

Kevin Yamauchi

ALL RIGHTS RESERVED 


\section{COMMITTEE MEMBERSHIP}

TITLE:

Prediction of Articular Cartilage Remodeling During Dynamic Compression with a Finite Element Model

AUTHOR:

Kevin Yamauchi

DATE SUBMITTED: June 2012

COMMITTEE CHAIR: Stephen Klisch, Ph.D.

COMmitTeE MEMBER: Andrew Davol, Ph.D.

COMMITTEE MEMBER: Scott Hazelwood, Ph.D. 


\begin{abstract}
Prediction of Articular Cartilage Remodeling During Dynamic Compression with a Finite Element Model
\end{abstract}

Kevin Yamauchi

First, an in vitro growth experiment was performed to test the hypothesis that applying dynamic unconfined compression during culture produces heterogeneous remodeling in newborn bovine articular cartilage explants. Heterogeneous measures of cartilage microstructure were obtained by biochemical assays and quantified polarized light microscopy. Significant differences were measured between the GAG content in the inner and outer portions of the samples stimulated with dynamic unconfined compression. The COL fiber network was found to be more highly aligned in the inner portion of the sample than in the peripheral region.

Next, a poroelastic finite element model with a remodeling subroutine was developed to test the hypothesis that the magnitude of relative interstitial fluid velocity and maximum principle strain stimulate GAG and COL fiber network remodeling, respectively, in articular cartilage during culture with dynamic unconfined compression. The GAG remodeling law was successful in predicting the heterogeneous changes in GAG content. The collagen remodeling law was not successful in predicting the changes in the COL network microstructural orientation, suggesting another mechanical cue is responsible for stimulating the remodeling of the COL fiber network. 


\section{Acknowledgements}

I would like to thank my advisors Dr. Stephen Klisch and Dr. Scott Hazelwood for their mentorship over the past three years. I feel lucky to have worked so closely with them and will always be grateful for the time they dedicated to this project.

Additionally, I would like to thank Dr. Robert Sah for his mentorship and guidance. Also, thank you Dr. Albert Chen and Dr. Greg Williams for helping me get acquainted with the wet lab.

I would also like to thank my labmates, Matt Griebel and Matteo Taffetani for sharing the frustrations of finite element analysis with me. Without you two, my sanity would be a mere speck in my rearview mirror.

Finally, I would like to thank my friends and family for supporting me and understanding when I had to work long hours.

This project was supported by grants from NSF and NIH. 


\section{Contents}

List of Tables viii

List of Figures ix

$\begin{array}{lll}1 & \text { Introduction } & 1\end{array}$

2 Background Review 5

2.1 Articular Cartilage Constituents . . . . . . . . . . . . . . . 5

2.2 Biphasic Articular Cartilage Models . . . . . . . . . . . . . . . 6

2.3 Glycosaminoglycan Remodeling . . . . . . . . . . . . . . . 6

2.4 Collagen Remodeling . . . . . . . . . . . . . . . . . . . . 7

2.5 Quantitative Polarized Light Microscopy . . . . . . . . . . . . 7

\begin{tabular}{lll}
\hline 3 & Theory & 8
\end{tabular}

3.1 Kinematics . . . . . . . . . . . . . . . . . . . 8

3.2 Biphasic Theory . . . . . . . . . . . . . . . . . . . . . . . 10

3.3 Constitutive Stress Equations . . . . . . . . . . . . . . . . . . 12

3.4 Collagen Fiber Distribution Function . . . . . . . . . . . . . . 18

3.5 Glycosaminoglycan Remodeling . . . . . . . . . . . . . . . . 22

3.6 Collagen Remodeling . . . . . . . . . . . . . . . . . . . . . 24

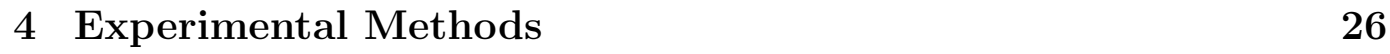

4.1 Harvest . . . . . . . . . . . . . . . . . . . . . . 26

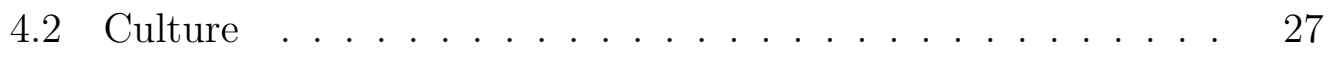

4.3 Biochemical Analysis . . . . . . . . . . . . . . . . . . . . . 28

4.4 q

4.5 Statistical Analysis . . . . . . . . . . . . . . . . . . . 30 
4.6 Untreated Control Group Biochemical Data . . . . . . . . 30

$\begin{array}{lll}5 & \text { Implementation } & 32\end{array}$

5.1 User Material Definition Subroutine . . . . . . . . . . . . . . . 32

5.2 Remodeling Subroutine . . . . . . . . . . . . . . . . . . . 33

5.3 Mesh and Boundary Conditions . . . . . . . . . . . . 33

5.4 Material Properties . . . . . . . . . . . . . . . . . . 34

5.5 Anisotropic Collagen Fiber Distribution . . . . . . . . . . . 35

5.6 Simulation Steps $\ldots \ldots \ldots$

5.7 Solver Parameters . . . . . . . . . . . . . . . . . . . . . . . . . 39

5.8 Post-Processing $\ldots \ldots \ldots \ldots \ldots$

5.9 Parameter Determination $\ldots \ldots \ldots$. . . . . . . . . 40

5.10 Computational Requirements . . . . . . . . . . . 40

$\begin{array}{lll}6 & \text { Results } & 42\end{array}$

$6.1 \quad$ Experimental Results . . . . . . . . . . . . . . . . . . . . . 43

$6.2 \quad$ Finite Element Analysis Results . . . . . . . . . . . . . . 45

\begin{tabular}{lll}
\hline 7 & Discussion & 49
\end{tabular}

Bibliography 54 


\section{List of Tables}

4.1 Table of experimental groups . . . . . . . . . . . . . . . . . . . 27

5.1 Table of initial material properties. Note that $\alpha_{a v g}, \alpha_{s t . d e v .}$, and $\lambda$ are the parameters used to define $R(\Theta, \Phi)$. $\alpha_{\text {st.dev. }}$ is the standard deviation of the average fiber angles in the ROI, which is defined as $\sigma^{2}$ where $\sigma$ is variance. See Section $|3.4|$ for details. 35

6.1 GAG remodeling algorithm parameters . . . . . . . . . . . . . 45

6.2 COL remodeling algorithm parameters . . . . . . . . . . . . . 48 


\section{List of Figures}

3.1 Initial deformation to reach the stress-free solid matrix reference configuration .................... . . 14

3.2 A unit sphere is defined at each material point to describe the continuous distribution of collagen fibers. . . . . . . . . . . 16

3.3 The unit circle at the intersection of the plane defined by the intersection $\Phi=\Phi_{0}$ and the unit sphere describes the distribution of collagen fibers in the plane of the qPLM image. . . . . . . . 19

3.4 A sample collagen fiber area fraction distribution showing the contributions of $F_{G}$ and $F_{N G} . \ldots \ldots . \ldots . . \ldots 21$

4.1 The composition of the the inner (gray) and outer (white) region of each sample was analyzed separately. The inner region was removed with a $1.5 \mathrm{~mm}$ diameter dermal punch. . . . . . . . . 29

4.2 qPLM fields of view . . . . . . . . . . . . . . . . . . . 30

5.1960 element mesh of the quarter-disk model. . . . . . . . . . . 34

5.2 Simulation steps for six days of growth. . . . . . . . . . . . . 36

5.3 The initial swelling balances the constituent stresses to reach a stress-free equilibrium reference configuration . . . . . . . 37

$6.1 \quad$ Effect of dynamic unconfined compression on GAG content . . 43

6.2 Effect of dynamic unconfined compression on COL content. . . 44

6.3 Predicted GAG density compared to experimental data. . . . . 45

6.4 Predicted COL distribution after 6 days of simulated growth. $\quad 46$

6.5 Predicted changes in COL fiber network with only the algorithm to predict remodeling due to chemical stimuli active. . . . . . 47 


\section{Chapter 1}

\section{Introduction}

Articular cartilage (AC) is a low-friction, load bearing soft tissue that lines the ends of long bones. Healthy joints allow for pain-free ambulation. AC allows the joints to articulate smoothly and helps absorb joint loads [1]. When $\mathrm{AC}$ accumulates damage, its ability to facilitate smooth, pain-free joint articulation is reduced and in some cases, entirely compromised. AC can be damaged by disease, repetitive loading or traumatic injury [1,2]. As of 2003, arthritis had affected approximately 46 million people in the United States of America and cost 81 billion dollars in medical treatments [3]. AC has a poor capacity for natural repair due to its avascular nature and low metabolic activity [4]. For severe injuries, surgical intervention is the only repair strategy. Surgical therapies include marrow stimulation therapies, e.g. microfracture, autologous chondrocyte implantation, osteochondral graft implantation, and in cases of extensive degeneration, total joint replacement [4 6]. These therapies are expensive, invasive and do not guarantee a full restoration of joint function $[4,7]$. 
Tissue engineering has been proposed as a viable alternative to current repair strategies [8,9]. Tissue engineered AC could replace damaged cartilage tissue in order to restore normal joint function and prevent the onset of progressive degeneration. Tissue engineering methods could be used to create new tissue or alter existing tissue. For small defects, only the effected area would be repaired. In cases of extensive damage, the whole joint surface could be replaced. It may be critical to control the geometry, mechanical properties, and tribological properties of engineered tissues in order to develop a consistent surgical grafting technique.

$\mathrm{AC}$ has been shown to have highly complex mechanical properties through in vitro mechanical testing. $\mathrm{AC}$ exhibits nonlinear stress-strain behavior, tension-compression asymmetry, and anisotropic mechanical properties 10 12. AC constituents have distinct mechanical roles. The glycosaminoglycans (GAGs) are primarily responsible for resisting compressive loads and the collagen (COL) fiber network resists tensile and shear loads. In vitro studies of the mechanical properties of $\mathrm{AC}$ in compression have correlated compressive modulus with glycosaminoglycan and and water content and negatively correlated Poisson's ratio with collagen content [13 15]. Tensile modulus has been positively correlated with collagen and collagen-specific pyridinoline crosslink content 16. Understanding the structure-function relationship may be a key element in successfully engineering cartilage implants capable of surviving implantation and restoring normal joint function.

AC composition can be altered through both chemical and mechanical stimuli. Growth factors such as insulin-like growth factor 1 (IGF-1) and transforming growth factor beta 1 (TGF- $\beta 1$ ) have been used to promote geometric growth or alter the constituent balance $13,14,17,19$. Mechanical stimuli such 
as relative interstitial fluid velocity and mechanical cues such as maximum principle strain direction have been correlated with AC remodeling 2023. Previous studies have stimulated tissue by applying static and dynamic unconfined compression, dynamic shear, and permeation [20 26]. Determining how chemical and mechanical cues shape AC growth may facilitate engineered $\mathrm{AC}$ tissue that matches the geometric and mechanical properties of the natural tissue.

Finite element models are useful for studying the structure-function relationship of $\mathrm{AC}$ because they are capable of predicting the contributions of individual constituents [27]. It is difficult to isolate individual components during in vitro mechanical tests because removing a constituent affects the remaining constituents. For example, in immature tissue, removing GAG to study the COL network alters the tensile properties of the tissue [19]. Biphasic theory has been used to model the mechanical response of AC [28]. Models predicted large deformations due to physiological loading [29, 30], so finite deformation biphasic theory was developed [31. Bimodular biphasic models were developed in order to model the tension-compression asymmetry exhibited by AC [32]. Other models with anisotropic fiber-reinforced models have been developed to model the anisotropic distribution of COL fibers in $\mathrm{AC}$ tissue [33].

Dynamic unconfined compression at moderate strain amplitudes (1-4\%) and higher frequencies $(0.01-0.1 \mathrm{~Hz}$.) can increase biosynthesis [34]. As previously mentioned, the remodeling of GAG and the COL network has been associated with relative interstitial fluid velocity and maximum principle strain, respectively. For this reason, dynamic unconfined compression on a disk is an ideal load case for studying growth and remodeling because the the magnitude 
of the relative interstitial fluid velocity is dependent on radial position and the maximum principle strain is in the radial direction. A previous study correlated the heterogeneous changes in AC biosynthesis after applying dynamic unconfined compression to a biphasic finite element model. The model used had an isotropic solid matrix and did not include remodeling algorithms. Additionally, the final composition of the AC explant was not assessed [20]. This study aims to use an approach that integrates in vitro growth experiments and a finite element model with remodeling subroutines to study how AC explants remodel when stimulated with dynamic unconfined compression.

The hypotheses of this study were: (1) dynamic unconfined compression of $\mathrm{AC}$ explants in culture results in heterogeneous GAG and COL remodeling changes and (2) the magnitude of relative interstial fluid velocity and maximum principle strain stimulate remodeling of GAG and the COL fiber network, respectively, in articular cartilage during culture with dynamic unconfined compression. The objectives of this work were to: (A) perform in vitro growth experiments on newborn bovine AC explants in a custom bioreactor that applies dynamic unconfined compression during culture, (B) develop a novel AC remodeling finite element model with an isotropic GAG constituent and anisotropic collagen fiber network, and (C) use the results from objectives (A) and (B) to test hypothesis (2). 


\section{Chapter 2}

\section{Background Review}

\subsection{Articular Cartilage Constituents}

$\mathrm{AC}$ is composed of a porous solid matrix fully saturated with an interstitial

fluid. AC is primarily composed of water. Of the solid matrix constituents, COL and GAG are the most prevalent. [35]

Collagen is a protein found in tissues such as bone, cartilage, and tendon that forms a network of fibers that reinforces the tissue [36]. Collagen fibrils form bundles called fibers. In AC, these fibers form an organized structure that resists the swelling pressure of proteoglycans, shear stresses, and tensile stresses [35]. In mature articular cartilage, collagen fibrils are oriented perpendicular to the subchondral bone in the deep zone, parallel to the articular surface in the superficial zone, and isotropically distributed in the middle zone as the fibrils transition from the deep zone to the superficial zone [35].

Proteoglycans are composed of chains of GAG arranged in a bottlebrush configuration. The GAG's negative fixed charge density attracts and retains 
water within the cartilage tissue, which aids in the hydration and lubrication of the $\mathrm{AC}$ tissue. In addition, the negative fixed charge density creates a swelling pressure within the tissue that pre-stresses the collagen network and resists compressive loading 35].

\subsection{Biphasic Articular Cartilage Models}

Biphasic theory, which considers an intrinsically incompressible solid saturated with an incompressible fluid, has been used to successfully model the mechanical response of AC in compression [28]. Early biphasic models of biological tissues were based on biphasic theory developed by Craine, Green, and Nagdi [37 39]. A review of pioneering biphasic theories can be found in [40]. Large AC deformations were predicted for physiological loading [29, 30], so finite deformation biphasic theories were developed [31,41, 42]. The model used in this study builds upon these, adding a continuous distribution of collagen fibers 43 .

\subsection{Glycosaminoglycan Remodeling}

During maturation, the GAG concentration in AC remains roughly the same in order to maintain the compressive properties [44, 45]. In vitro growth experiments have shown that culturing AC in IGF-1 increases GAG content [13]. Additionally, it has been demonstrated that GAG synthesis increases under dynamic unconfined compression 21]. Through the use of a biphasic AC model, GAG synthesis was positively correlated with the relative interstitial fluid velocity during in vitro dynamic unconfined compression [20,46, 48]. 


\subsection{Collagen Remodeling}

The orientation of collagen fibrils in AC evolves during maturation. In immature cartilage, the fibrils tend to be oriented parallel to the articular

surface through the depth of tissue 49 51. As the cartilage matures, the fibrils in the deep zone reorient to a direction perpendicular to the subchondral bone 49 53. It has been proposed that the fibrils reorient toward a local preferred direction. Candidates for the determining the preferred direction axis include direction of maximum Cauchy stress, maximum tensile principle strain, and a function of the tensile principle strains [36, 54]. Experimental evidence suggests that this remodeling is a result of simultaneous tissue resorption and neoformation [55], but does not validate a particular mechanical trigger for collagen remodeling.

\subsection{Quantitative Polarized Light Microscopy}

Quatitative polarized light microscopy (qPLM) has been used to assess the orientations of collagen fibrils in $\mathrm{AC}$. $\mathrm{AC}$ is a birefringent material due to the cylindrical shape of the collagen fibers. Using methods described in [56], AC samples can be imaged on a polarized light microscope and then the resulting birefringent signal analyzed to assess the orientations of the collagen fibers. 


\section{Chapter 3}

\section{Theory}

\subsection{Kinematics}

Consider a continuous body $B$, which occupies a reference configuration $\boldsymbol{\kappa}_{0}(B)$ at time $\mathrm{t}=0$. At some time $\mathrm{t}, B$ occupies a new space known as the current configuration $\boldsymbol{\kappa}(B)$.

The material point $P_{0}$ on $B$ is located by the position vector $\mathbf{X}$ in $\boldsymbol{\kappa}_{0}(B)$. In the current configuration the same point on $B$, now known as $P$, is described by the position vector $\mathbf{x}$. Thus the motion of the material points on the body $B$ can be described by the mapping

$$
\mathbf{x}=\chi(\mathbf{X}, t)
$$

The displacement vector of each material point on $B$ from the reference to current configuration is

$$
\mathbf{u}=\mathbf{x}-\mathbf{X}
$$


The deformation gradient tensor $\mathbf{F}$ is

$$
\mathbf{F}=\frac{\partial \mathbf{x}}{\partial \mathbf{X}}
$$

The right Cauchy-Green deformation tensor $\mathbf{C}$ is related to the deformation gradient tensor by

$$
\mathbf{C}=\mathbf{F}^{T} \mathbf{F}
$$

The Lagrangian strain tensor $\mathbf{E}$ is

$$
\mathbf{E}=\frac{1}{2}(\mathbf{C}-\mathbf{I})
$$

where $\mathbf{I}$ is the identity tensor.

The change in the volume of $B$ from the reference to the current configuration is described by the Jacobian $J$, which is

$$
J=\operatorname{det}(\mathbf{F})
$$

where $\operatorname{det}(\cdot)$ is the determinant operator.

The continuity equation is

$$
\dot{\rho}+\rho \operatorname{div}(v)=\rho c
$$

where $\operatorname{div}(\cdot)$ is the divergence operator and $\rho c$ is the mass added due to growth. Density in the current configuration $\rho$ is defined as

$$
J \rho=\rho_{0}+\int r_{0} d t
$$

where $\rho_{0}$ is the density in the reference configuration and $r_{0}$ is the time rate of change of density. In this study, the time scale of remodeling was assumed to be much larger than that of the mechanical loading, so growth was calculated 
once a day. For this incremental growth problem from time $t_{0}$ to $t_{1}$, the current density at time $t_{0} \leq t<t_{1}$ is

$$
J_{1} \rho_{1}=\rho_{0}
$$

At time $t=t_{1}$ the sample is unloaded, so $J=1$ and the remodeling algorithm is applied. The current configuration at $t=t_{1}$ is

$$
\rho_{1}=\rho_{0}+r_{0} \Delta t
$$

where $\Delta t=t_{1}-t_{2}$. The density in the current configuration after $n$ increments of growth is

$$
\rho_{n}=\rho_{0}+r_{0} \Delta t_{0}+r_{1} \Delta t_{1}+\ldots+r_{n} \Delta t_{n}
$$

\subsection{Biphasic Theory}

Finite deformation biphasic theory has been used to model AC. The basic theory is summarized here. In biphasic theory, the tissue is modeled as a material composed of solid and fluid phases. In this summary, variables pertaining to the solid and fluid phases will be denoted by a superscript $s$ and $f$, respectively. The volume fractions of the solid and fluid phases obey the saturation condition

$$
\phi^{s}+\phi^{f}=1
$$

where $\phi^{s}$ and $\phi^{f}$ are the volume fractions of the solid fluid phases, respectively. The volume fraction of constituent $m$ is

$$
\phi^{m}=\frac{\rho^{m}}{\rho_{T}^{m}}
$$

where $\rho^{m}$ and $\rho_{T}^{m}$ are the apparent and true densities, respectively, of the constituent $m$. Apparent density is the constituent mass divided by the tissue 
volume and true density is the constituent mass divided by the constituent volume.

Using equation 3.12 and the incompressibility assumption, the continuity equation becomes

$$
\operatorname{div}\left(\phi^{s} \mathbf{v}^{s}+\phi^{f} \mathbf{v}^{f}\right)=0
$$

where $\mathbf{v}$ is the absolute velocity vector of the constituent.

The relative interstitial fluid velocity is the velocity of the fluid constituent relative to the solid constituent and is

$$
\mathbf{v}^{f / s}=\mathbf{v}^{f}-\mathbf{v}^{s}
$$

The total Cauchy stress of the tissue is defined as

$$
\boldsymbol{\sigma}=\boldsymbol{\sigma}^{s}+\boldsymbol{\sigma}^{f}
$$

Both constituent stresses are defined as per tissue area. ABAQUS uses an equivalent stress measure, effective stress, which is defined as

$$
\overline{\boldsymbol{\sigma}}^{*}=\overline{\boldsymbol{\sigma}}+\chi u_{w} \mathbf{I}
$$

where $\overline{\boldsymbol{\sigma}}$ is the effective stress on the porous solid matrix $\left(\boldsymbol{\sigma}^{s}\right), \chi$ is the saturation, $u_{w}$ is the pore pressue $\left(\boldsymbol{\sigma}^{f}\right)$, and $\mathbf{I}$ is the identity tensor. Saturation is the fraction of the pore volume filled with interstitial fluid and therefore can range from 0 to 1 . In this biphasic theory, the tissue is assumed to be fully saturated, so $\chi=1$.

The void ratio $e$ is the ratio of the volume fraction of the fluid phase to the volume fraction of the solid phase. The porosity, $n$, is defined as

$$
n=\frac{e}{1+e}
$$


Permeability, $k$, is the measure of the resistance to fluid flow through the solid matrix. The material permeability for 1-D strain, as derived in 41 is

$$
k=k_{0}\left[\frac{\phi_{0}^{s} \phi^{f}}{\left(1-\phi_{0}^{s}\right) \phi^{s}}\right]^{\kappa} \exp \left[M\left(\frac{\lambda^{2}-1}{2}\right)\right]
$$

where $k_{0}$ and $\phi_{0}^{s}$ are the material permeability and solid phase volume fraction, respectively, in the zero-strain configuration. $M$ is a non-dimensional permeability coefficient that describes the exponential increase in permeability with strain and $\lambda$ is the stretch of the material. $\kappa$ is a positive constant that controls the rate at which permeability reaches zero as the volume fraction of the solid phase approaches 1 . Similar to equation $(3.8)$, the continuity equation is

$$
\phi^{s}=\frac{\phi_{0}^{s}}{J}
$$

Inserting 3.12 and the definition of the void ratio into equation 3.20 , the Jacobian $J$ can be expressed as

$$
J=\frac{1+e}{1+e_{0}}
$$

Using equation 3.21, equation 3.19 can be written in terms of the void ratio as

$$
k=k_{0}\left[\frac{e}{e_{0}}\right]^{2} \exp \left[\frac{M}{2}\left(\left(\frac{1+e}{1+e_{0}}\right)^{2}-1\right)\right]
$$

\subsection{Constitutive Stress Equations}

Abaqus requires all stress to be reported as Cauchy stress. Additionally, for finite strain simulations, Abaqus requires the exact consistent Jacobian matrix in order to guide the solver to the correct solution. The formulation for the exact consistent Jacobian matrix was developed in 43, 57 and is presented 
below in indicial notation.

$$
\begin{aligned}
\mathbb{C}_{i j k l}^{J A C}= & \frac{1}{J}\left\{\left[\left(\delta_{i k} \delta_{p l}+\delta_{p k} \delta_{i l}\right) \boldsymbol{\sigma}_{p j}+\frac{J}{2}\left(\delta_{j k} \delta_{n l}+\delta_{n k} \delta_{j l}\right) \boldsymbol{\sigma}_{i n}\right]\right. \\
& \left.+\left[\overline{\mathbb{C}}_{A B C D} F_{i A} F_{j B} F_{k C} F_{l D}\right]\right\}
\end{aligned}
$$

where $\delta$ is the Kroenecker delta, $\boldsymbol{\sigma}$ is the solid matrix Cauchy stress, and $\overline{\mathbb{C}}$ is the material elasticity tensor. The material elasticity tensor is defined as

$$
\overline{\mathbb{C}}=\frac{\partial \mathbf{S}}{\partial \mathbf{E}}
$$

where $\mathbf{S}$ is the second Piola-Kirchhoff stress tensor and $\mathbf{E}$ is the Lagrangian strain tensor. Second Piola-Kirchoff is related to Cauchy stress by

$$
J \boldsymbol{\sigma}=\mathbf{F S F}^{T}
$$

where $\mathbf{F}^{T}$ is the transpose of the deformation gradient tensor.

The model's solid matrix is composed of three constituents. The GAG constituent accounts for the internal swelling pressures created by the negative fixed charge density of the GAG. The second constituent, COL, models the mechanical response of the tissue's collagen fiber network. Finally, the ground substance matrix (MAT) constituent accounts for the various matrix components not accounted for by the GAG and COL constituents. The total Cauchy stress of the solid matrix is the sum of the constituent stresses:

$$
\boldsymbol{\sigma}^{S M}=\boldsymbol{\sigma}^{G A G}+\boldsymbol{\sigma}^{C O L}+\boldsymbol{\sigma}^{M A T}
$$

Therefore, constituents can have non-zero stress when the tissue is in a zero stress state. Each of the three solid matrix constituents occupies an initial configuration $\kappa_{0}^{m}$ which must undergo an initial deformation $\mathbf{F}_{0}^{m}$ where $m$ is the constituent (Figure 3.1). 


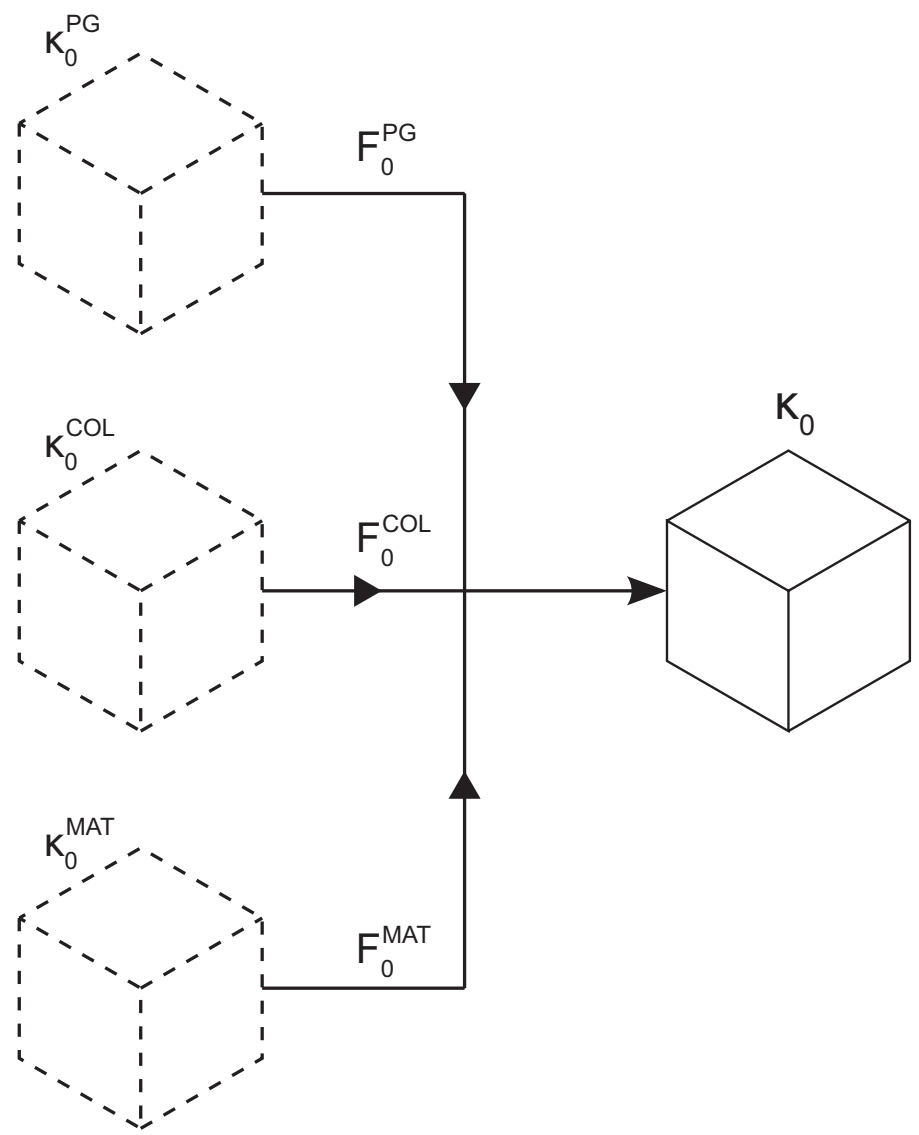

Figure 3.1: Each of the solid matrix constituents occupies an initial reference configuration and must undergo an initial deformation to satisfy the initial stress-free solid matrix reference configuration.

\subsubsection{Glycosaminoglycan Constituent Stress}

The formulation for GAG stress is based on the Poisson-Boltzmann model of a unit cell of molecule in an ionic solution and was developed in [43. The GAG constituent Cauchy stress tensor $\boldsymbol{\sigma}^{G A G}$ is described by

$$
\boldsymbol{\sigma}^{G A G}=-\alpha_{1}\left(\rho^{G A G}\right)^{\alpha_{2}} \mathbf{I}
$$


where $\rho^{P G}$ is the apparent density of GAG in the current configuration. Using equation (3.8), the GAG density in the current configuration is

$$
\rho^{G A G}=\frac{\rho_{0}^{G A G}}{J}
$$

where $\rho_{0}^{G A G}$ is the apparent GAG density in the reference configuration.

The following GAG stress coefficients provided a good fit $\left(R^{2}=0.98\right)$ to the Poisson-Boltzmann model developed by Buschmann and Grodzinsky 58 for GAG densities typically seen in immature bovine AC: $\alpha_{1}=2.87 \frac{\mathrm{N} \cdot \mathrm{mL} L^{2.5}}{\mathrm{~m}^{2} \cdot \mathrm{m} g^{2.5}}$ and $\alpha_{2}=2.5$ [43].

\subsubsection{Collagen Constituent Stress}

The material model for the collagen constituent was developed in [57] and is summarized here. In this material model, a continuous distribution of collagen fibers is defined at each material point. The collagen fiber distribution is described by a unit sphere discretized into pyramidal elements that are each composed of a specific volume fraction of collagen fibers (Figure 3.2). 


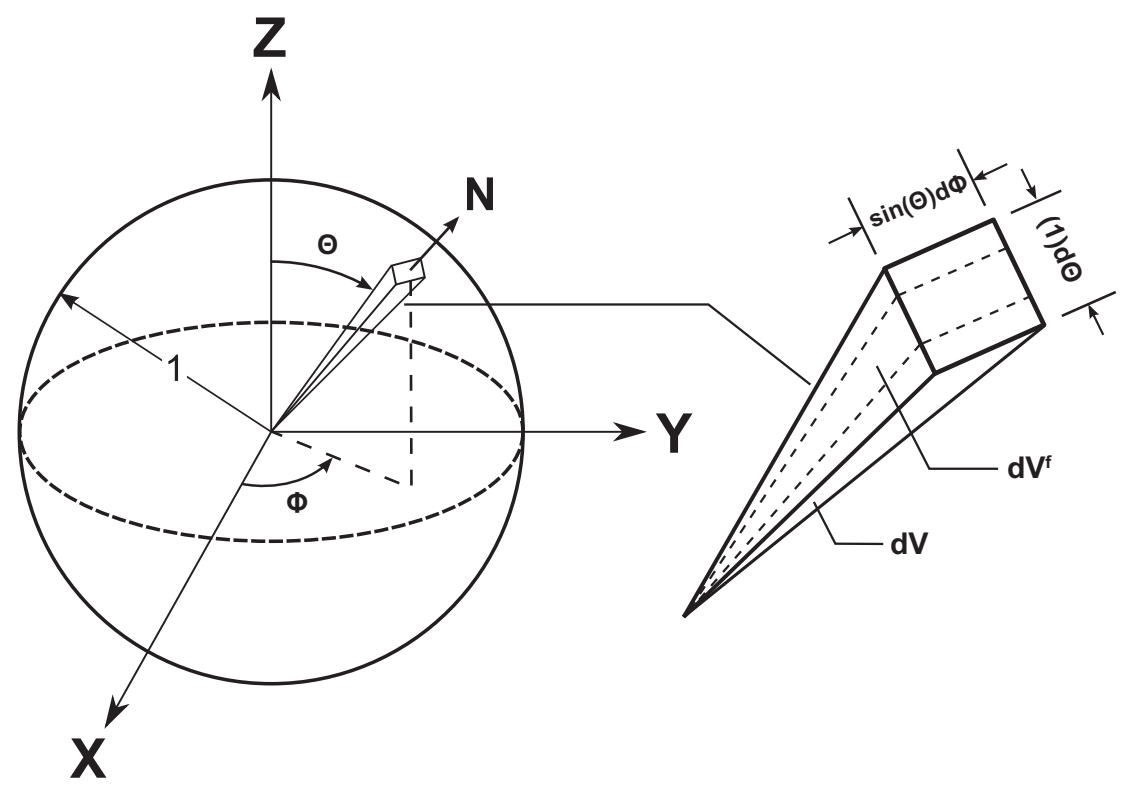

Figure 3.2: A unit sphere is defined at each material point to describe the continuous distribution of collagen fibers.

The total volume fraction of collagen in the unit sphere $\phi^{f}$ is

$$
\phi^{f}=\frac{V^{f}}{V}
$$

where $V^{f}$ is the volume of collagen fibers in the unit sphere and $V$ is the unit sphere volume. The differential volume $\mathrm{dV}$ is

$$
d V=\frac{1}{3} \sin \Theta d \Theta d \Phi
$$

The orientation of each pyramidal element is described by the outward normal vector $\mathbf{N}$. The collagen fibril volume fraction in the differential volume element with outward normal vector $\mathbf{N}$ is then

$$
\phi_{N}^{f}=\frac{d V_{N}^{f}}{d V}
$$

The continuous distribution of collagen fibers $R(\theta, \phi)$ is described by

$$
R(\theta, \phi)=\frac{\phi_{N}^{f}}{V}
$$


where $\mathrm{V}$ is the volume of the unit sphere. Integrating $\mathrm{R}$ over the volume gives the total collagen fiber volume fraction $\phi^{f}$

$$
\phi^{f}=\int_{V} R(\theta, \phi) d V
$$

The second Piola-Kirchhoff stress of the collagen constituent is given as

$$
\mathbf{S}^{C O L}=\int_{0}^{2 \pi} \int_{0}^{\pi} R(\theta, \phi) H E_{f} E_{N}(\mathbf{N} \otimes \mathbf{N}) \frac{1}{3} \sin \theta d \theta d \phi
$$

where $E_{f}$ is the true fiber modulus and $E_{N}$ is the 1-D Lagrangian strain in direction $\mathbf{N}$, which is described by

$$
E_{N}=\mathbf{N} \cdot \mathbf{E N}
$$

where $\mathbf{E}$ is the Lagrangian strain tensor as defined in equation (3.5). Since collagen fibers are only mechanically active in tension, the heavyside step function $\mathrm{H}$ deactivates fibers that are in compression. $\mathrm{H}$ is defined in terms of the Lagrangian strain in the direction, $E_{N}$ as

$$
H\left(E_{N}\right)= \begin{cases}1 & \text { for } E_{N}>0 \\ 0 & \text { for } E_{N} \leq 0\end{cases}
$$

\subsubsection{Ground Substance Matrix Constituent Stress}

The ground substance matrix constituent accounts for the various solid matrix constituents in articular cartilage that are neither collagen nor proteoglycans. A hyperelastic Neo-Hookean solid was chosen to model the ground substance matrix [4]. The second Piola-Kirchoff stress tensor of a Neo-Hookean solid is expressed as

$$
\mathbf{S}^{M A T}=\mu\left(\mathbf{I}-\mathbf{C}^{-1}\right)
$$

where $\mu$ is the shear modulus and $\mathbf{C}^{-1}$ is the inverse of the right Cauchy-Green deformation tensor. 


\subsubsection{Immobility Constraint}

It is assumed in this model that all of the constituents are bound to the solid matrix and are not allowed to move within the tissue. Therefore, all constituents experience the same deformation. That is,

$$
\mathbf{F}^{S M}=\mathbf{F}^{G A G}=\mathbf{F}^{C O L}=\mathbf{F}^{M A T}
$$

where $\mathbf{F}^{S M}$ is the solid matrix deformation gradient tensor relative to the solid matrix stress-free reference configuration and $\mathbf{F}^{G A G}, \mathbf{F}^{C O L}$, and $\mathbf{F}^{M A T}$ are the deformation gradient tensors of the GAG, COL, and MAT constituents relative to the solid matrix stress-free reference configuration, respectively.

\subsection{Collagen Fiber Distribution Function}

The collagen fiber volume fraction distribution function used in equation (3.34) is estimated from two-dimensional images analyzed with qPLM. The intersection of the image plane defined by $\Phi=\Phi_{0}$ and the unit sphere is the unit circle centered at $X_{0}$. Each unit circle captures the fibers in the plane $\Phi=\Phi_{0}$ that pass through $X_{0}$. The unit circle is divided into differential elements with differential area

$$
d A=\frac{1}{2} d \theta
$$

The directional area fraction of COL fibers in direction $\mathbf{N}$ is

$$
\gamma_{N}^{f}=\frac{d A^{f}}{d A}
$$

where $d A^{f}$ is the area of dA occupied by fibers. The total fiber area $A^{f}$ of the unit circle is

$$
A^{f}=\int_{A} \gamma_{N}^{f} d A
$$




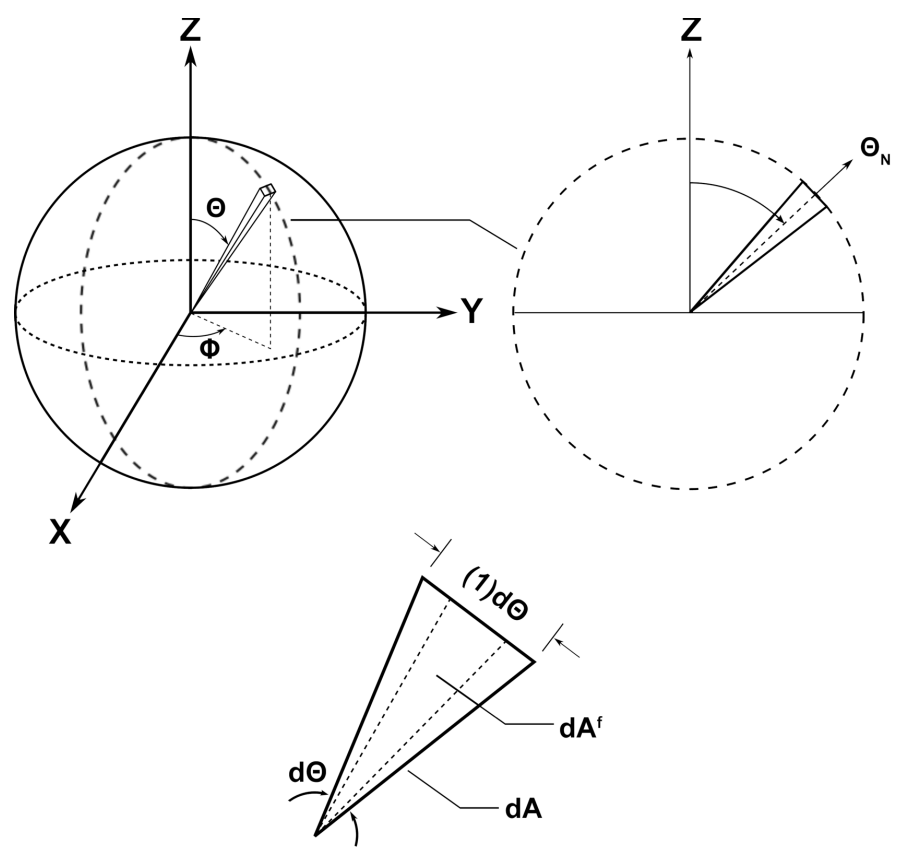

Figure 3.3: The unit circle at the intersection of the plane defined by the intersection $\Phi=\Phi_{0}$ and the unit sphere describes the distribution of collagen fibers in the plane of the qPLM image.

The area fraction of fibers in direction $\mathbf{N}$ is $d A^{f}$ normalized by $A^{f}$. Combining equations (3.40) and (3.41), the area fraction of fibers in direction $\mathrm{N}$ is

$$
\frac{d A_{N}^{f}}{A^{f}}=\frac{\gamma_{N}^{f} d A}{\int_{A} \gamma_{N}^{f} d A}
$$

The area fraction is subject to the constraint

$$
\frac{1}{A^{f}} \int_{A} d A_{N}^{f} d A=1
$$

As shown in Figure 3.3, the differential triangle element $d A$ in direction $\mathbf{N}$ bisects the differential pyramid element dV in direction $\mathbf{N}$. Assuming Delesse's principle is valid for these differential elements, the area fraction and volume fraction in direction $\mathbf{N}$ are equal,

$$
\gamma_{N}^{f}=\phi_{N}^{f}
$$


Now consider a finite triangle element with mean direction $\Theta_{N}$ and range $\Delta \Theta_{N}$. The area of the element is

$$
\Delta A_{N}=\frac{1}{2} \Delta \Theta_{N}
$$

where $\Delta$ denotes a finite quantity. All finite triangle elements are assumed to be homogeneous. The unit semi-circle is discretized into $m$ elements with $\Theta_{N}$ ranging from $-\frac{\pi}{2}$ to $\frac{\pi}{2}$. Equations 3.42 and 3.43 become

$$
\frac{\Delta A_{N}^{f}}{A^{f}}=\frac{\gamma_{N}^{f} \Delta A_{N}}{\sum_{m} \gamma_{N}^{f} \Delta A_{N}}
$$

and

$$
\sum_{m} \frac{\Delta A_{N}^{f}}{A^{f}}=1
$$

Using equation (3.44), equation (3.46) becomes

$$
\frac{\Delta A_{N}^{f}}{A^{f}}=\frac{\phi_{N}^{f} \Delta A_{N}}{\sum_{m} \phi_{N}^{f} \Delta A_{N}}
$$

The value of $\frac{\Delta A_{N}^{f}}{A^{f}}$ for each element is estimated from qPLM by fitting a distribution to the histogram of average fiber angles from analysis of the birefringent signal. When an image is analyzed with qPLM, each pixel is assigned an average fiber angle $\alpha$ based on the birefringent signal. The pixels are then grouped into regions of interest, for which a histogram of the $\alpha$ values from each pixel in the region of interest is generated.

The distribution of fibers is modeled as a Gaussian distribution $F_{G}$ superposed with a background isotropic distribution $F_{N G}$ that ensures all elements have a minimum area fraction to aid convergence (Figure 3.4. See the discussion section for justification for the background isotropic distribution. The area fraction distribution is

$$
\frac{\Delta A_{N}^{f}}{A^{f}}=C F_{G}\left(\Theta_{N}\right)+F_{N G}\left(\Theta_{N}\right)
$$


where $\mathrm{C}$ is a scalar constant to enforce constraint (3.47).

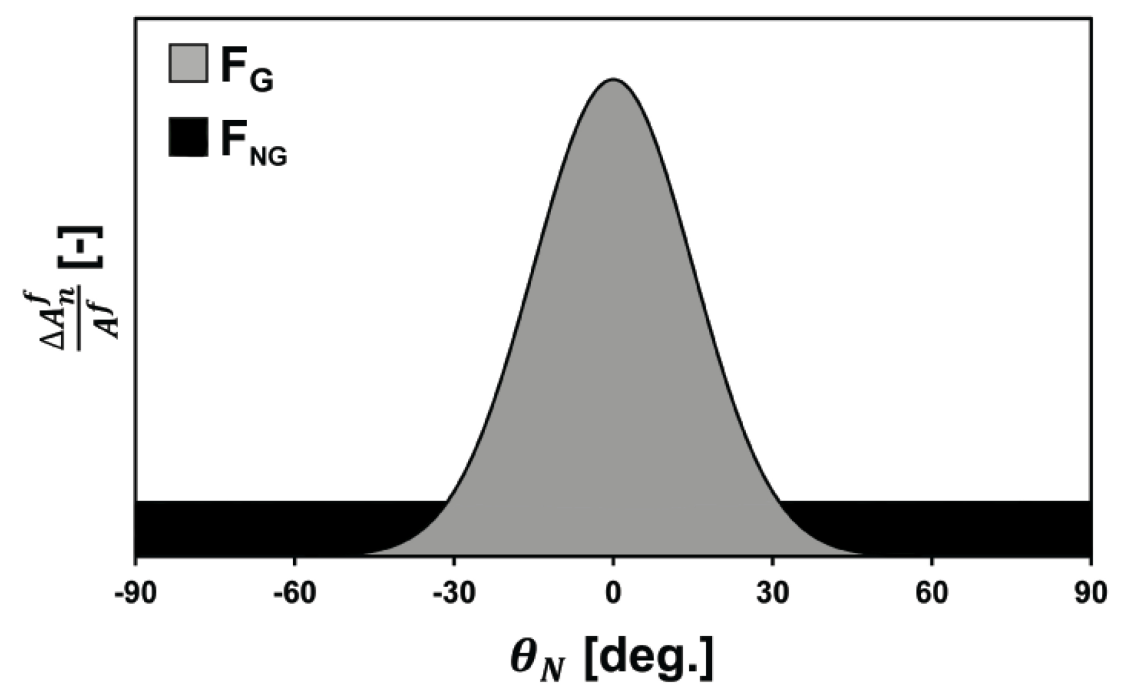

Figure 3.4: A sample collagen fiber area fraction distribution showing the contributions of $F_{G}$ and $F_{N G}$.

The Gaussian distribution function $f$ of $x$ is defined as

$$
f(x)=\frac{e^{-(x-\mu)^{2} / 2 \sigma^{2}}}{\sqrt{2 \pi \sigma^{2}}}
$$

where $\mu$ is the mean of $x$ and $\sigma^{2}$ is the standard deviation of $x$. In order to satisfy the constraint (3.43), $f(x)$ must be divided by $\int f(x) d x=1$. The normalized distribution function $f^{\prime}(x)$ is

$$
f^{\prime}(x)=\frac{e^{-(x-\mu)^{2} / 2 \sigma^{2}}}{\int e^{-(x-\mu)^{2} / 2 \sigma^{2}} d x}
$$

Equation (3.4) was adapted for use with a unit circle from [59].In this application,

$$
\begin{aligned}
f^{\prime}(x) & =F_{G}\left(\Theta_{N}\right) \\
x & =\Theta_{N} \\
\mu & =\alpha_{\text {mean }} \\
d x & =\frac{1}{2} d \Theta
\end{aligned}
$$


where $\alpha_{\text {mean }}$ is the mean value of the $\alpha$ values for the region of interest. Applying 3.52 to equation and integrating from 0 through $\pi$ yields

$$
F_{G}\left(\Theta_{N}\right)=\frac{e^{-\left(\Theta_{N}-\mu\right)^{2} / 2 \sigma^{2}}}{\int_{0}^{\pi} e^{-(\beta-\mu)^{2} / 2 \sigma^{2}} d \beta}
$$

The distribution function $F_{N G}\left(\Theta_{N}\right)$ is defined as

$$
F_{N G}\left(\Theta_{N}\right)= \begin{cases}\lambda-F_{G}\left(\Theta_{N}\right) & \text { for } F_{G}\left(\Theta_{N}\right)<\lambda \\ 0 & \text { for } F_{G}\left(\Theta_{N}\right) \geq \lambda\end{cases}
$$

where $\lambda$ is the value of the area fraction of the background isotropic distribution.

Substituting equation (3.53) into equation (3.49) gives the area fraction distribution function

$$
\frac{\Delta A_{N}^{f}}{A^{f}}\left(\Theta_{N}\right)=C\left(\frac{e^{-\left(\Theta_{N}-\mu\right)^{2} / 2 \sigma^{2}}}{\int_{0}^{\pi} e^{-(\beta-\mu)^{2} / 2 \sigma^{2}} d \beta}\right)+F_{N G}\left(\Theta_{N}\right)
$$

The scalar constant C must be solved for numerically because $F_{G}\left(\Theta_{N}\right)$ and $F_{N G}\left(\Theta_{N}\right)$ are coupled.

The distribution of area fractions calculated in equation (3.55) can be used in equation (3.48) to create a system of $m$ equations and $m+1$ unknowns ( $m$ values of $\phi_{N}^{f}$ and $\left.\sum_{m} \phi_{N}^{f} \Delta A_{N}\right)$. This indeterminacy is solved by applying the constraint (3.33) with an experimental measure of $\phi^{f}$. The COL fiber volume fraction distribution function $R(\Theta, \Phi)$ can then be defined from the values of $\phi_{N}^{f}$ using equation 3.32).

\subsection{Glycosaminoglycan Remodeling}

The GAG remodeling was separated into two types: remodeling due to chemical stimuli and remodeling due to mechanical stimuli. The change in 
GAG density in the reference configuration $\rho_{0}^{G A G}$ is

$$
\frac{d \rho_{0}^{G A G}}{d t}=\left(\frac{d \rho_{0}^{G A G}}{d t}\right)^{c}+\left(\frac{d \rho_{0}^{G A G}}{d t}\right)^{m}
$$

where the $c$ and $m$ superscripts denote remodeling due to chemical and mechanical stimuli, respectively.

The change in GAG density due to chemical stimuli accounts for the change in GAG density observed after free-swelling culture in IGF-1. The rate of change in GAG density was assumed to be constant. Therefore, the rate of change in GAG density due to chemical stimuli is

$$
\left(\frac{d \rho^{G A G}}{d t}\right)^{c}=\tau
$$

where $\tau$ is a scalar constant that describes the rate of change in GAG density due to chemical stimuli.

The change in GAG density due to mechanical stimuli accounts for the effect of dynamic unconfined compression on the change in GAG density. The synthetic rate of aggrecan in dynamically compressed is proportional to the relative interstitial fluid velocity [20]. Therefore, the rate of change in GAG density due to mechanical stimuli is

$$
\frac{d \rho_{0}^{G A G}}{d t}=\kappa \times v^{f / s}
$$

where $v^{f / s}$ is the relative interstitial fluid velocity magnitude, $\times$ signifies scalar multiplication, and

$$
\kappa\left(v^{f / s}\right)= \begin{cases}\beta & \text { for } v_{\text {max }}^{f / s}>v_{r}^{f / s} \\ 0 & \text { for } v_{\text {max }}^{f / s} \leq v_{r}^{f / s}\end{cases}
$$

where $\beta$ is a scalar constant and $v_{r}^{f / s}$ is the relative interstitial fluid velocity magnitude threshold for GAG densification. Substitute equations (3.57) and 
(3.58) into equation 3.56 to get the total rate of change in GAG density

$$
\frac{d \rho^{G A G}}{d t}=\tau+\kappa v^{f / s}
$$

\subsection{Collagen Remodeling}

As with GAG remodeling, a separate COL remodeling law was proposed for the remodeling due to chemical stimuli and remodeling due to mechanical stimuli.

$$
\frac{d \phi_{N}^{f}}{d t}=\left(\frac{d \phi_{N}^{f}}{d t}\right)^{c}+\left(\frac{d \phi_{N}^{f}}{d t}\right)^{m}
$$

The change in COL volume fraction due to chemical stimuli accounts for the change in collagen volume fraction and fiber distribution after culture in IGF1. Since the fiber distribution is assumed not change during free-swelling culture in IGF-1, a scaling growth law was chosen, so the overall COL fiber volume fraction is reduced, but the fiber distribution is maintained. The rate of change in collagen volume fraction in direction $\mathbf{N}$ is

$$
\left(\frac{d \phi_{N}^{f}}{d t}\right)^{c}=\Upsilon \phi_{N}^{f}
$$

where $\Upsilon$ is a scalar constant that modulates the rate at which COL is resorbed or deposited during free-swelling IGF-1 culture.

The exact mechanical trigger for the remodeling of the collagen fibril network is not known. As previously stated, it has been proposed that COL fibers reorient towards a local preferred direction. The exact mechanism for determining the local preferred direction is unknown, but the direction of maximum tensile stress and the direction of maximum tensile strain have been proposed. The following growth law was proposed, which increases the collagen volume 
fraction in direction $\mathbf{N}$ when the fiber strain is greater than a threshold value.

$$
\left(\frac{d \phi_{N}^{f}}{d t}\right)^{m}=\kappa\left(E_{N}\right) \times p h i_{N}^{f}
$$

where $\times$ indicates scalar multiplication and

$$
\kappa\left(E_{N}\right)= \begin{cases}\eta & \text { for } E_{N, \text { max }}>E_{N, g} \\ 0 & \text { for } E_{N, \text { max }} \leq E_{N, g}\end{cases}
$$

$\eta$ is the rate the collagen fiber volume fraction is increased in the direction $\mathbf{N}$ when the fiber strain trigger is exceeded, $E_{N, \max }$ is the maximum Lagrangian fiber strain in the direction $\mathbf{N}$, and $E_{N, g}$ is the fiber strain threshold that triggers an increase in COL fiber volume fraction. Substitute equations 3.63 and 6.3 into equation 3.61 to get the total rate of change of the COL fiber volume fraction in direction $\mathbf{N}$

$$
\frac{d \phi_{N}^{f}}{d t}=\Upsilon \phi_{N}^{f}+\kappa\left(E_{N}\right) \times p h i_{N}^{f}
$$




\section{Chapter 4}

\section{Experimental Methods}

An in vitro growth study was designed and performed to test the hypothesis that dynamic unconfined compression leads to heterogeneous growth and remodeling of the GAG and COL constituents. The objective was to design a bioreactor capable of applying dynamic unconfined compression to articular cartilage during in vitro culture and provide experimental data to validate and calibrate the proposed growth laws.

\subsection{Harvest}

Full thickness blocks of articular cartilage were harvested from the ridge of the patellofemoral groove of fresh bovine knees from newborn (approximately 1-3 week old) calves. $2.5 \mathrm{~mm}$ diameter cores were extracted from the blocks and cut down to $1 \mathrm{~mm}$ thick disks with the articular surface intact using a custom cutting fixture. 
All samples were allowed to free-swell in phosphate-buffered saline. After free-swelling, geometric measurements were taken in sterile conditions. Disk thickness was measured with a laser micrometer, wet weight was measured with an electronic balance, and disk diameter was assessed optically with a digital single lens reflex camera and a custom MATLAB script. After geometric measurements were taken, the samples were divided into experimental groups. The samples with the most uniform thickness were chosen to be in the dynamically loaded group. The experimental groups can be seen below in Table 4.1.

Table 4.1: Table of experimental groups. D0 is the untreated control group, FSB is the free-swelling in medium $+\mathrm{IGF}-1$, and $\mathrm{S} 10+\mathrm{D}$ was treated with dynamic unconfined compression.

\begin{tabular}{|c|c|c|c|c|c|}
\hline$\#$ & Name & Growth Factor & Treatment & Duration & Days Culture \\
\hline 0 & D0 & - & - & - & - \\
\hline 1 & FSB & IGF-1 & FS & 24 hours - on & 6 \\
\hline 2 & S10+D & IGF-1 & S10+D & 8 hours - on & 6 hours - off \\
\hline
\end{tabular}

\subsection{Culture}

A custom bioreactor was designed to apply dynamic unconfined compression to the samples during culture. The bioreactor had eight wells for loaded samples and four wells for free-swelling (unloaded) samples. A heat exchanger in the base of the bioreactor maintained an internal temperature of $37 \mathrm{deg}$. Celsius. The bioreactor was machined out of a single block of polysophone. 
Polysophone was chosen for biocompatibility and compatibility with the autoclave. The displacement was applied by a Dynastat uniaxial load frame. The dynamically loaded samples were loaded continuously with a static offset of $10 \%$ compressive strain with a sinusoidal displacement of $2 \%$ amplitude and a frequency of $0.10 \mathrm{~Hz}$. for 8 hours per day for 6 days. After loading, the samples were allowed to free swell for the remaining 16 hours of the day. Free-swelling controls were incubated in the free-swelling wells of the bioreactor. All samples were cultured in medium (Dulbeccos modified Eagles medium with $100 \mu \mathrm{g} / \mathrm{ml}$ ascorbate, $0.01 \%$ bovine serum albumin, $0.1 \mathrm{mM}$ nonessential amino acids, 0.4 mM l-proline, $2 \mathrm{mM}$ l-glutamine, $10 \mathrm{mM}$ HEPES, $100 \mathrm{U} / \mathrm{ml}$ penicillin, 100 $\mu \mathrm{g} / \mathrm{ml}$ streptomycin, and $0.25 \mu \mathrm{g} / \mathrm{m}$ amphotericin B) with $50 \mathrm{ng} / \mathrm{mL}$ IGF-1. A full media change was performed at 3 days. No contamination was observed in any of the samples. After culture, geometric measurements were taken with the same methods as described in Section 4.1 .

\subsection{Biochemical Analysis}

A $1.5 \mathrm{~mm}$ diameter section was removed from the center of each cultured sample with a dermal punch (Figure 4.1). The inner and outer portions of the sample were analyzed separately to assess the radial dependence of the growth and remodeling. GAG mass was measured using the technique outlined in 60]. Collagen mass was assessed using [61]. GAG and COL densities were reported as constituent mass normalized to initial and final wet weight. COL

volume fraction was calculated by assuming the true density of COL to be $1.436 \mathrm{~g} / \mathrm{cm}^{3} 62$. The data for samples that were divided into inner and outer 
portions were not normalized to initial wet weight because they were sectioned after culture, so the initial wet weights were not known.

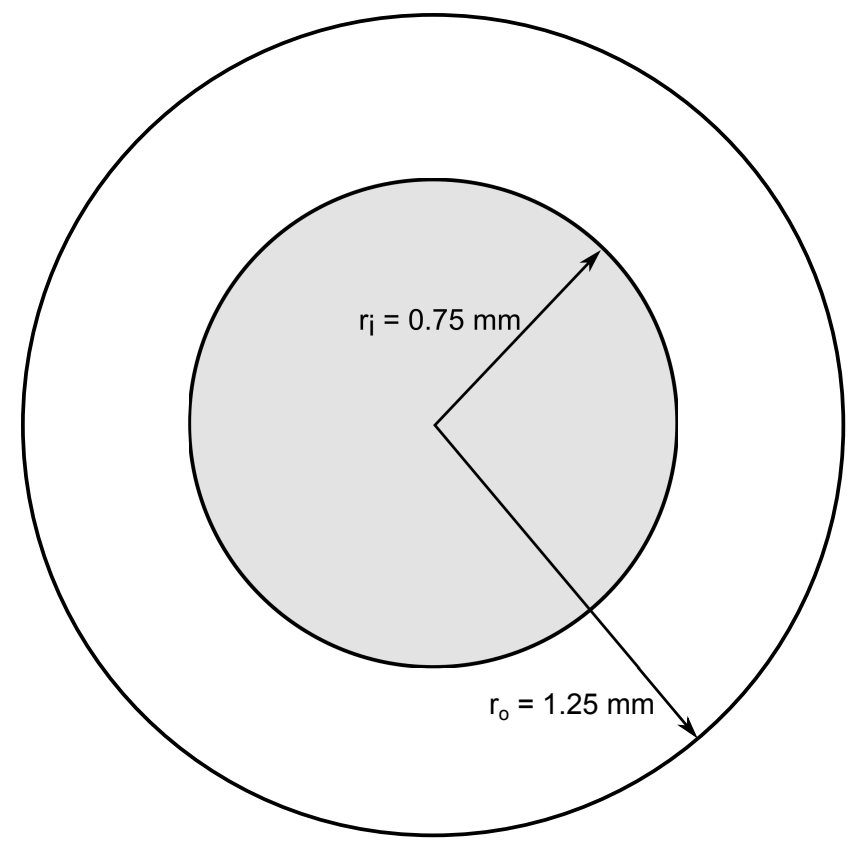

Figure 4.1: The composition of the the inner (gray) and outer (white) region of each sample was analyzed separately. The inner region was removed with a $1.5 \mathrm{~mm}$ diameter dermal punch.

\section{4 qPLM}

Four intact samples from the dynamic unconfined compression group were imaged with quantified polarized light microscopy (qPLM). The samples were cut in half in the sagittal plain. 5 micron thick slices were taken en face and placed on positively charged microscope slides. Each sample was imaged with four fields of view. The birefringence images were post-processed in MATLAB to determine the average fiber angle for each pixel in the image. The pixels were grouped into regions of interest that spanned the full thickness of the sample and had a width of approximately 200 microns. Then the mean and 
standard deviation of the average fiber angles in the ROI were calculated. A schematic of the fields of view and regions of interest is below in Figure 4.2 .

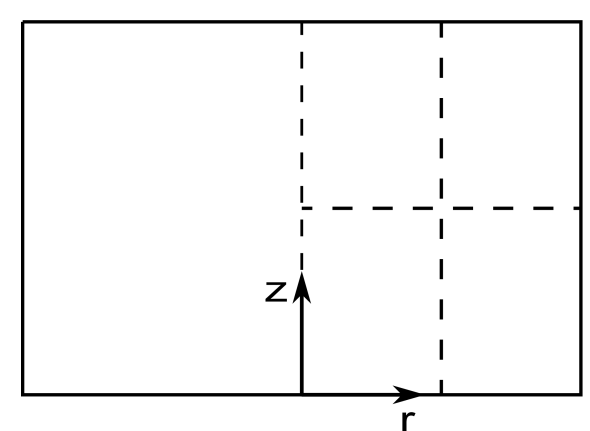

A

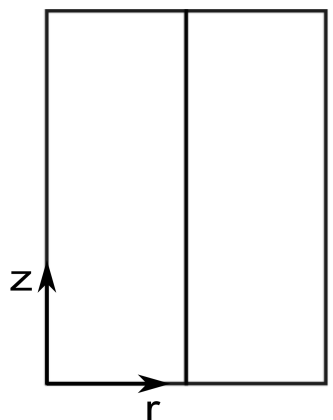

B

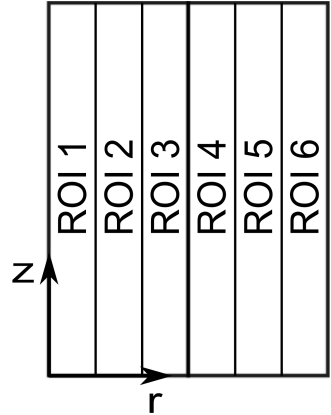

C

Figure 4.2: The full thickness of the sample was imaged in four fields of view (A). The vertically-aligned images were merged, so there were two images for each sample (B). The birefringent signal was analyzed for each of the images created in (B). Results were binned into six regions of interest with ROI 1 at $r=0$ and ROI 6 at the periphery of the sample $(\mathrm{C})$.

\subsection{Statistical Analysis}

Statistical differences between treatment and location for biochemical data were assessed with multiway ANOVA with post-hoc Tukey test. Statistical differences between the variances of the average fiber angle in different regions of interests in the qPLM data were assessed with multiway F-tests.

\subsection{Untreated Control Group Biochemical Data}

Due to an error in the biochemical assays, we were unable to use the biochemical data from the untreated controls group. AC from the patellofemoral groove has demonstrated consistent GAG and COL densities in previous stud- 
ies 13, 14, 17, 18. Biochemical data from [14 was used as it is the most recent and most closely matches the sites harvested from for this study. 


\section{Chapter 5}

\section{Implementation}

\subsection{User Material Definition Subroutine}

The user material definition subroutine (UMAT) used in this study is based on the one previously developed in [43] and 63]. The previous UMAT implemented an isotropic COL fiber distribution, a GAG constituitve stress equation based on the Poisson-Boltzman unit cell model, and an isotropic MAT material. This UMAT was updated with an anisotropic COL fiber distribution and the biochemical parameters (GAG density and COL fiber distribution) can now be updated during the simulation to allow for remodeling. Consequently, GAG density $\rho^{G A G}$ and the values of the COL fiber volume fraction distribution function $R(\theta, \phi)$ are now stored as a state variables, so they can can be updated by the remodeling subroutine. 


\section{$5.2 \quad$ Remodeling Subroutine}

The User Defined Fields Subroutine (USDFLD) was implemented to execute the remodeling subroutines. The USDFLD subroutine is called at the beginning of each increment. During the unconfined compression steps, the remodeling subroutine records the maximum fiber strain and relative interstitial fluid velocity at each integration point. During the remodeling steps, the remodeling subroutine uses the previously recorded maximum fiber strain and relative interstitial fluid velocity values with the remodeling laws to determine how the tissue remodeled. The remodeling subroutine calculated the remodeling at each integration point using the recorded maximum fiber strain for each finite triangle element and relative interstitial fluid velocity values once per simulated day. The remodeling was not calculated continuously because the time scale of growth (days) is much larger than that of the mechanical stimuli (seconds) 64.

\subsection{Mesh and Boundary Conditions}

The mesh used in this study was previously developed in 63]. Symmetry was used to reduce the disk to a quarter-disk. The mesh was created by seeding the radial edges and the height with 10 nodes each, resulting in 960 elements (Figure 5.1). Trilinear coupled stress-pore pressure elements were used (C3D8P). 


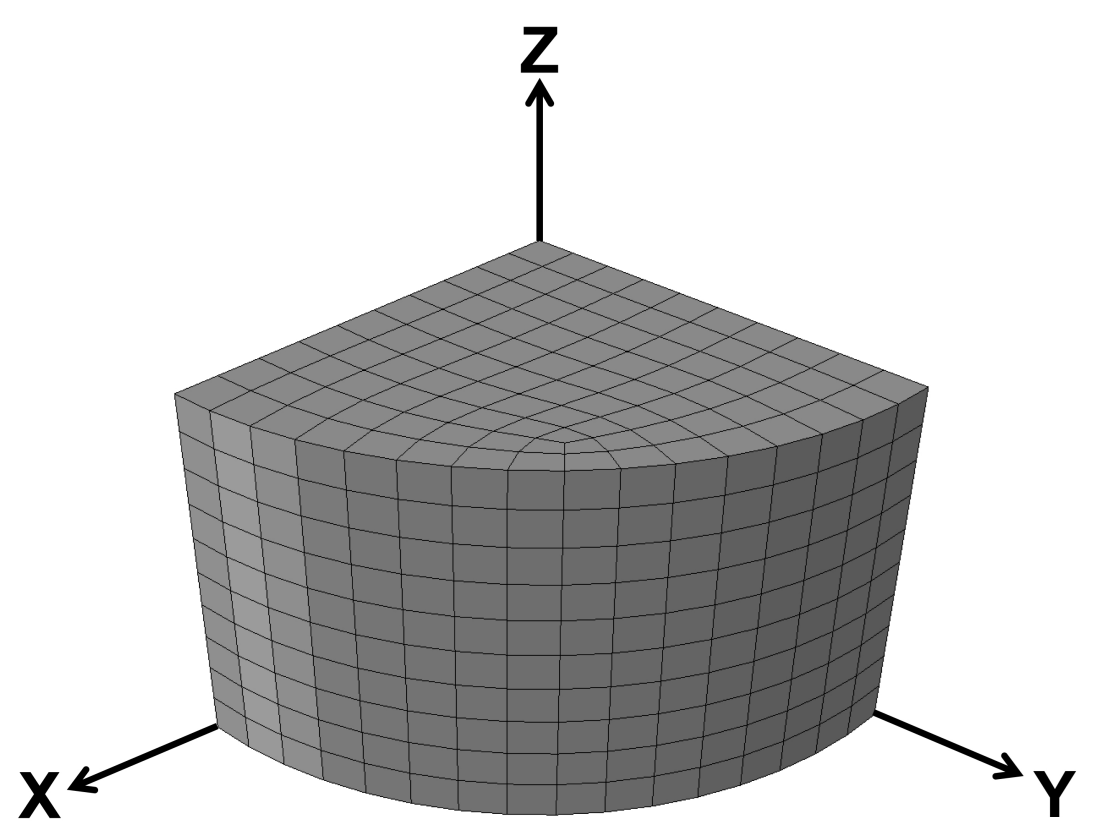

Figure 5.1: 960 element mesh of the quarter-disk model.

The faces with outward normal $(-1,0,0),(0,-1,0)$, and $(0,0,-1)$ were constrained in the direction of the outward normal. A pore pressure of 0 MPa was prescribed on the curved surface of the disk. All displacements were applied to the top surface.

\subsection{Material Properties}

The UMAT requires the true fiber modulus of COL $E_{f}$, the shear modulus of the MAT constituent $\mu$, the initial GAG density $\rho_{0}^{G A G}$, and the COL fiber volume fraction of each pyramidal element in the unit sphere. $E_{f}$ and the parameters for the initial $R(\Theta, \Phi)$ were obtained from [27], $\mu$ was obtained from [26], and $\rho_{0}^{G A G}$ was obtained from [14]. The material properties are below in Table 5.1. 
Table 5.1: Table of initial material properties. Note that $\alpha_{\text {avg }}, \alpha_{\text {st.dev. }}$, and $\lambda$ are the parameters used to define $R(\Theta, \Phi)$. $\alpha_{\text {st.dev. }}$ is the standard deviation of the average fiber angles in the ROI, which is defined as $\sigma^{2}$ where $\sigma$ is variance. See Section 3.4 for details.

\begin{tabular}{|c|c|c|c|c|c|}
\hline$E_{f}$ & $\mu$ & $\rho_{0}^{G A G}$ & $\alpha_{\text {mean }}$ & $\alpha_{\text {st.dev. }}$ & $\lambda$ \\
\hline $224.4 \mathrm{MPa}$ & $0.11 \mathrm{MPa}$ & $3.70 \% \mathrm{WW}$ & $0^{\circ}$ & $14.3^{\circ}$ & 0.002543 \\
\hline
\end{tabular}

\subsection{Anisotropic Collagen Fiber Distribution}

The unit sphere at each integration point was discretized into 1600 pyramidal elements $(\Delta \theta=4.5, \Delta \phi=4.5 \mathrm{deg})$. The initial volume fraction for each pyramidal element was calculated with a MATLAB implementation of the collagen fiber distribution function developed in Section 3.4. All integration points were assumed to have the same initial COL fiber volume fraction distribution. The average and standard deviations of the average fiber angle were from [27].

\subsection{Simulation Steps}

This simulation was modeled after the experiment outlined in Chapter 4. The input file was created in Abaqus/CAE and executed remotely through the command line. All steps implemented non-linear geometry due to the large deformations. See Figure 5.2 for the order of the simulation steps. 


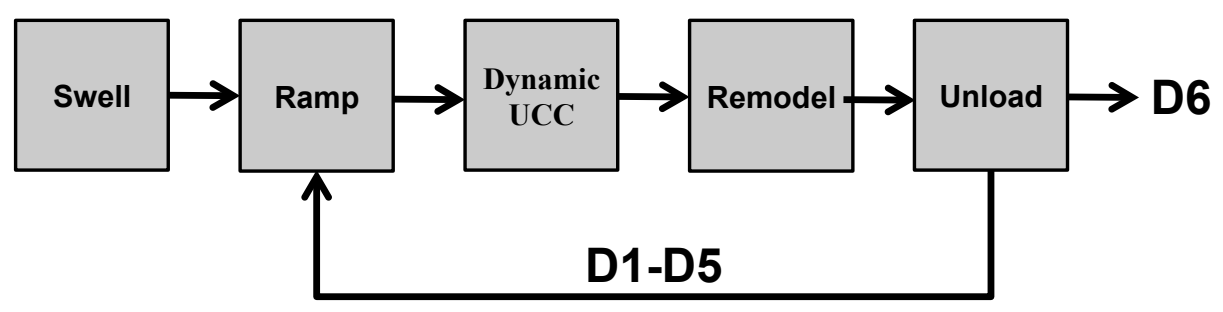

Figure 5.2: Simulation steps for six days of growth.

\subsubsection{Free-Swelling}

The stress balance between constituents leads to nonzero constituent stresses in the solid matrix stree-free reference configuration. The compressive (negative) swelling stress from the GAG constituent is balanced mostly by a tensile (positive) COL constituent stress and to a lesser extent, a tensile MAT constituent stress. During this step, Abaqus solves for the balance between constituent stresses such that the solid matrix is at stress-free equilibrium (Figure 5.3). The direct elastic solver is used, so pore fluid stress is not taken into account during the Free-Swelling step. 


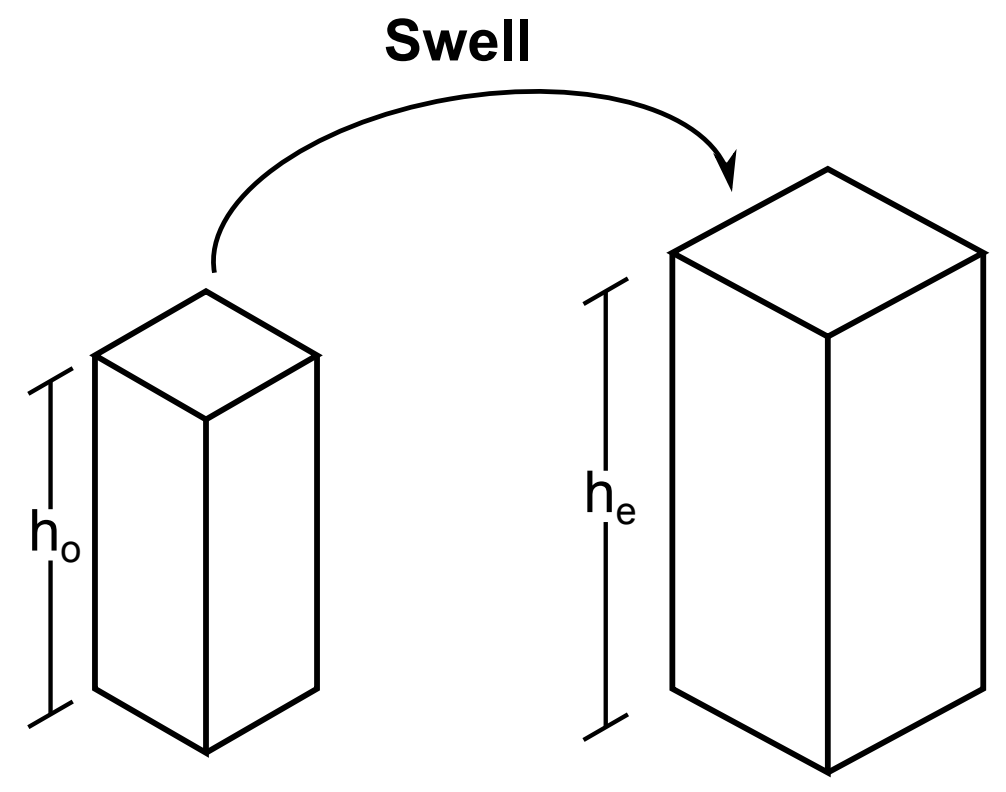

Figure 5.3: The Abaqus solver determines the deformation required for the COL and MAT constituents to balance the GAG constituent's compressive swelling stress in a single elastic step to satisfy the stress-free solid matrix reference configuration. The original height $h_{0}$ is the height defined by the initial model geometry. The equillibrium height $h_{e}$ is used to calculate axial strain in the following steps.

The equilibrium configuration is the stress-free reference configuration and is used to calculate strain, i.e. $h_{e}$ is used to calculate axial strain in subsequent steps.

\subsubsection{Ramp}

During the ramp step, the sample was compressed in unconfined compression to $10 \%$ strain, calculated from the previously determined $h_{e}$. Experimentally, the sample was compressed to $10 \%$ strain at a displacement rate of $v=0.001 \frac{\mathrm{mm}}{\mathrm{s}}$ and then allowed to reach equilibrium for 1200 seconds before dynamic unconfined compression was applied. Since in the experiment, the sample was allowed to reach static equilibrium at $10 \%$ compressive strain, the 
direct elastic solver was used during the Ramp step. Using the elastic solver reduces the computational time required for the Ramp step and makes a stress relaxation step unnecessary, saving additional computational time.

\subsubsection{Dynamic Unconfined Compression}

During the dynamic unconfined compression step, a sinusoidal displacement was applied at a frequency of $0.1 \mathrm{~Hz}$. with an amplitude of $2 \%$ strain and a static offset of $10 \%$ strain. The displacement is applied for 5 cycles (10 seconds) because the quarter-disk reaches steady state by the fifth cycle 63]. The SOILS solver was used in this step. The USDFLD subroutine was used to record maximum fiber strain for each pyramidal element and maximum relative interstitial fluid velocity at each integration point.

\subsubsection{Remodeling}

During the remodeling step, the remodeling subroutine in the USDFLD subroutine is executed. This is done during a single elastic step.

The remodeled GAG density at time $t+\Delta t$ is

$$
\rho_{0}^{G A G}(t+\Delta t)=\rho_{0}^{G A G}(t)+\left[\alpha+\kappa v^{f / s}(t)\right] \Delta t
$$

The remodeled COL fiber volume fraction in direction $\mathbf{N}$ at time $t+\Delta t$ is

$$
\phi_{N}^{f}(t+\Delta t)=\phi_{N}^{f}(t)+\left[C \phi_{N}^{f}(t)+\kappa\left(E_{N}\right)\right] \Delta t
$$




\subsubsection{Unloading}

The Unloading step is performed at the end of the simulation. The displacement boundary condition on the top of the disk is removed and the quarter disk is allowed to find equilibrium. Due to the heterogeneity of the disk after remodeling, loss of convergence was common during this step. In order to aid convergence, the magnitude of the displacement boundary condition on the top surface was slowly reduced.

\subsection{Solver Parameters}

The quasi-Newton solver with standard tolerances was used for all steps. The quasi-Newton solver uses a series of approximations of the Jacobian matrix, rather than calculating an exact solution [65]. Loss of convergence and long computational times were experienced when using the Full-Newton solver. This is likely due to the high anisotropic nature of the solid matrix and the size of the problem.

\subsection{Post-Processing}

All post-processing was performed through the Abaqus Python environment. Scripts were written to extract the field output values from the output database for integration points. Linear shape functions were then applied to interpolate values for points of interest. 


\subsection{Parameter Determination}

The remodeling parameters were determined iteratively. The GAG parameters were iterated for first. The parameter for remodeling due to chemical stimulus $\alpha$ was calculated by comparing the D0 GAG content and the D6 FSB GAG content from the experimental data and assuming that the GAG content had changed linearly with time. Pilot simulations were run to determine how the magnitude of the relative interstitial fluid velocity depended on radial position. Then the values of the parameters for GAG remodeling due to mechanical stimulus were predicted based on the radial relative interstitial fluid velocity magnitude profile and optimized.

After the GAG remodeling parameters were determined, the COL parameters were determined with a same method. First the parameters for the COL remodeling were calculated based on the experimental biochemical data. Next, simulations were run to determine the best parameters for the remodeling due to mechanical stimuli.

\subsection{Computational Requirements}

All simulations were run on a computer with an 8-core, 3.07 GHz Intel Core i7 processor and 8 GB of RAM. Simulations had a run time of approximately 2 days. The direct poroelastic solver cannot be multi-threaded, so only one core was used during steps with permeability active.

Each simulation created an output database that took approximately 60 GB of hard drive space. The file size was due to the large number of elements and state variables in the model. The large file size made post-processing re- 
sults through Abaqus/CAE inefficient. Instead, custom post-processing scripts were written in Python and executed through the Abaqus Python environment. 


\section{Chapter 6}

\section{Results}

The results from in vitro growth experiments and finite element models are below. All data are presented as mean \pm 1 standard deviation. 


\subsection{Experimental Results}

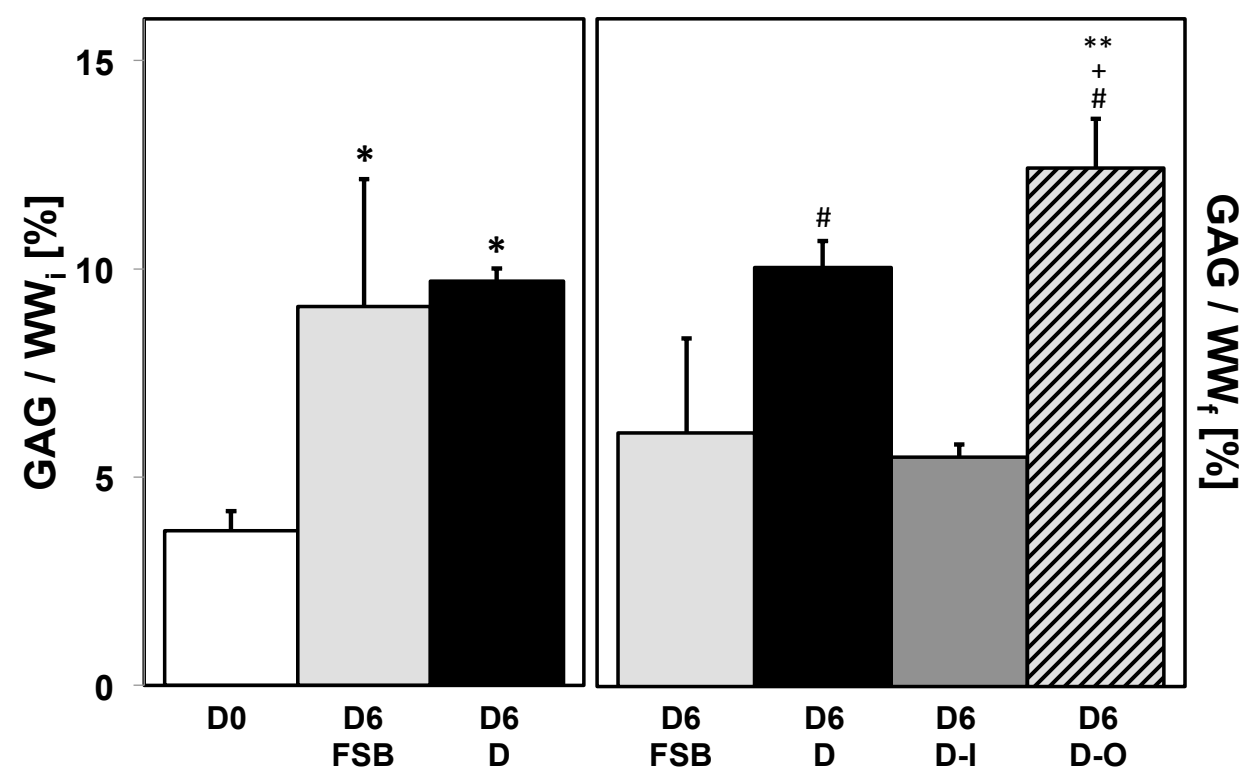

Figure 6.1: Effect of dynamic unconfined compression on GAG content. D0 indicates no treatment; D6 FSB indicates 6 days freeswelling culture; D6 D, D6 D-I, and D6 D-O indicate 6 days culture with dynamic unconfined compression for the intact specimen, inner region, and outer region, respectively. All samples cultured in medium supplemented with IGF-1. $\mathrm{p}<0.05$ when compared to $* \mathrm{D} 0$, \#D6 FSB, +D6 D, **D6 D-I 


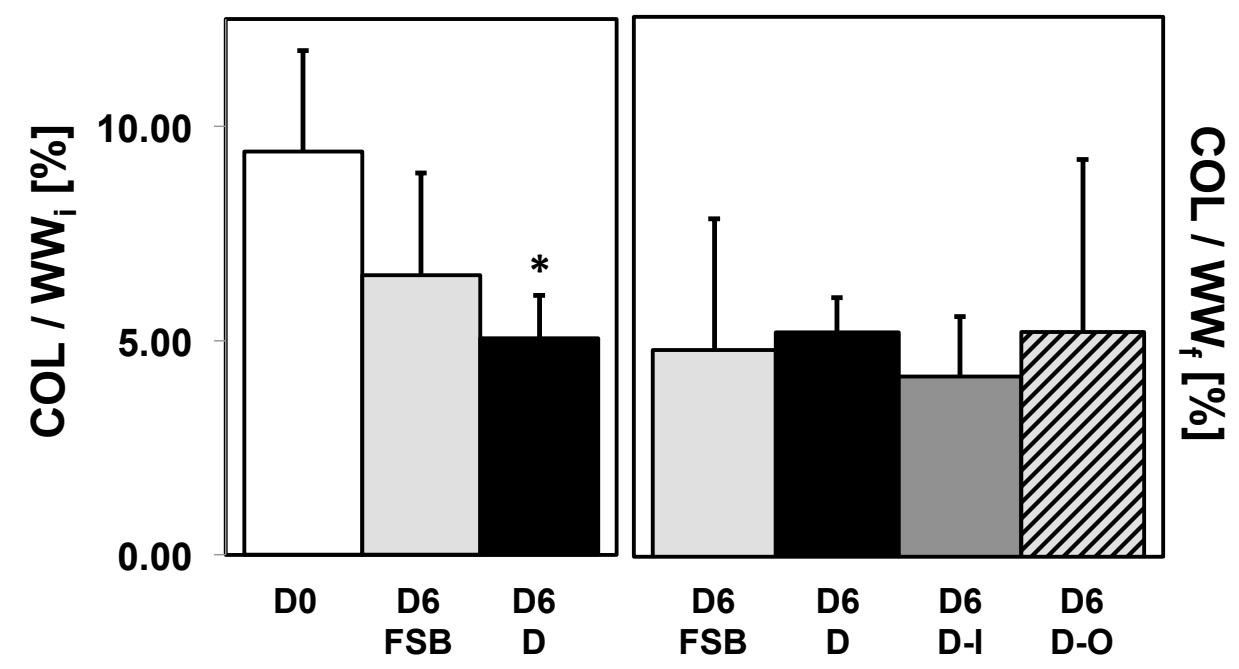

Figure 6.2: Effect of dynamic unconfined compression on COL content. D0 indicates no treatment; D6 FSB indicates 6 days freeswelling culture; D6 D, D6 D-I, and D6 D-O indicate 6 days culture with dynamic unconfined compression for the intact specimen, inner region, and outer region, respectively. All samples cultured in medium supplemented with IGF-1. $\mathrm{p}<0.05$ when compared to $* \mathrm{D} 0$ 


\subsection{Finite Element Analysis Results}

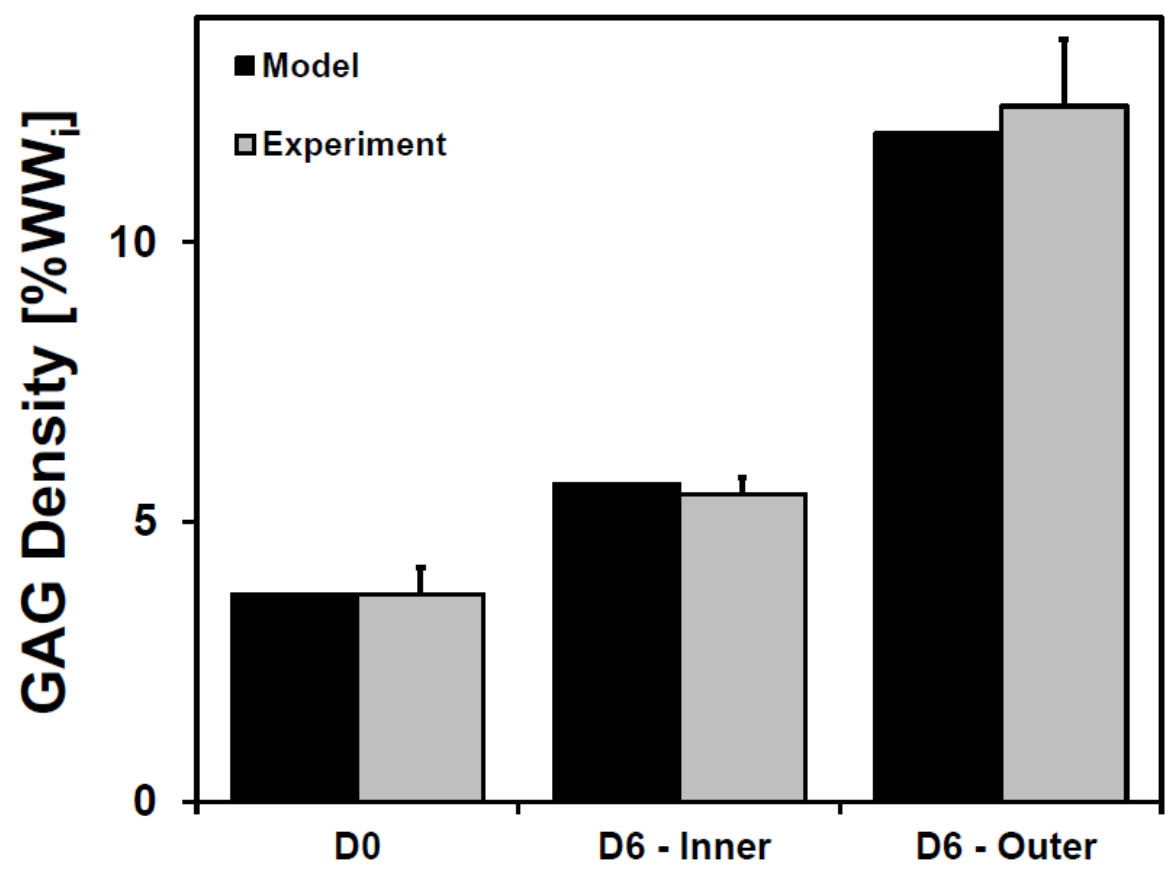

Figure 6.3: Predicted GAG density compared to experimental data. The predicted values are within $5 \%$ of the experimental data.

Table 6.1: Table of the final GAG remodeling law parameters.

\begin{tabular}{|c|c|c|}
\hline$\tau$ & $\beta$ & $v_{r}^{f / s}$ \\
\hline $0.2967 \frac{\left(\frac{m g}{m L}\right)}{d a y}$ & $9500 \frac{\frac{m g}{m L} / \frac{m m}{s}}{d a y}$ & $0.60 \mu \mathrm{m} / \mathrm{s}$ \\
\hline
\end{tabular}

For convenience, the GAG remodeling law is listed below.

$$
\frac{d \rho^{G A G}}{d t}=\tau+\kappa v^{f / s}
$$

where

$$
\kappa\left(v^{f / s}\right)= \begin{cases}\beta & \text { for } v_{\text {max }}^{f / s}>v_{r}^{f / s} \\ 0 & \text { for } v_{\text {max }}^{f / s} \leq v_{r}^{f / s}\end{cases}
$$


ROI 1
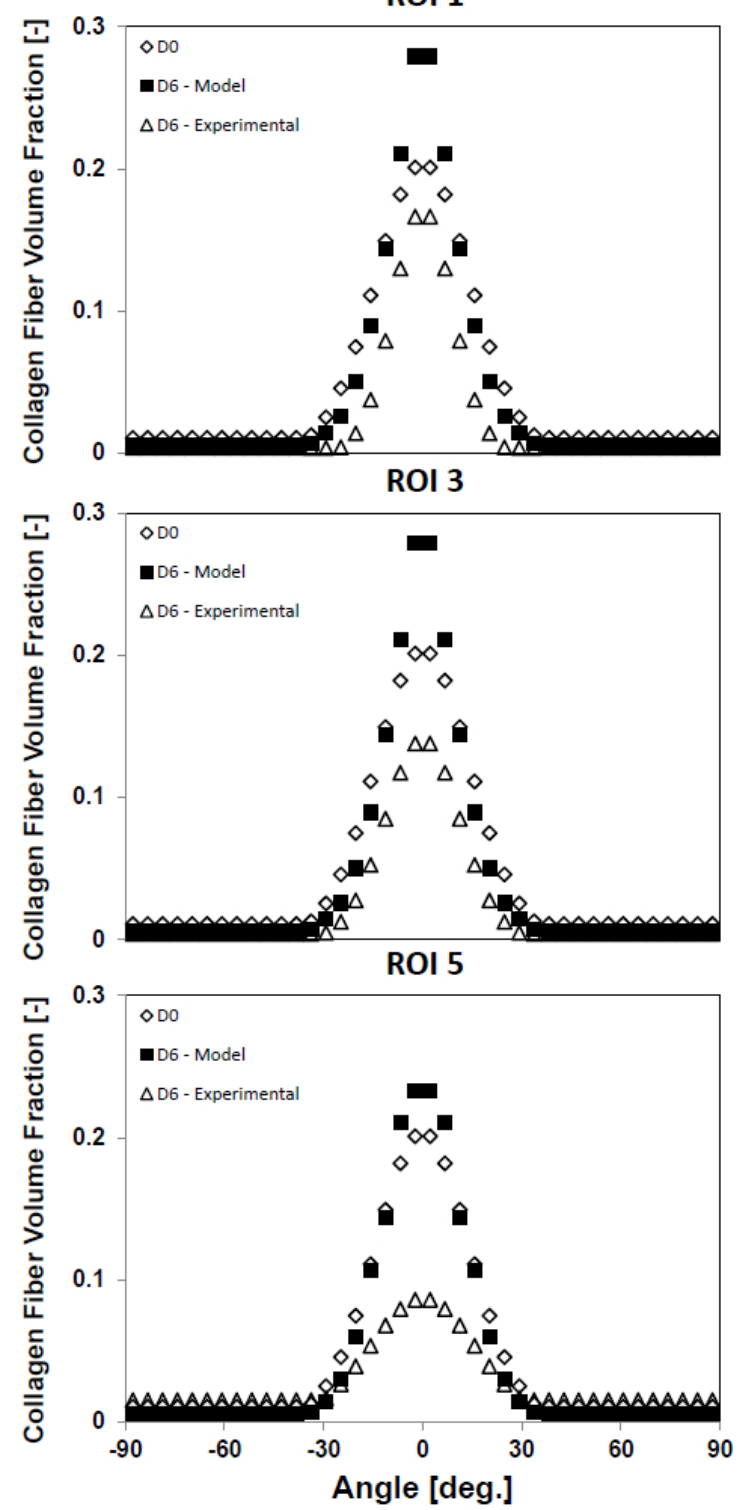

ROI 2
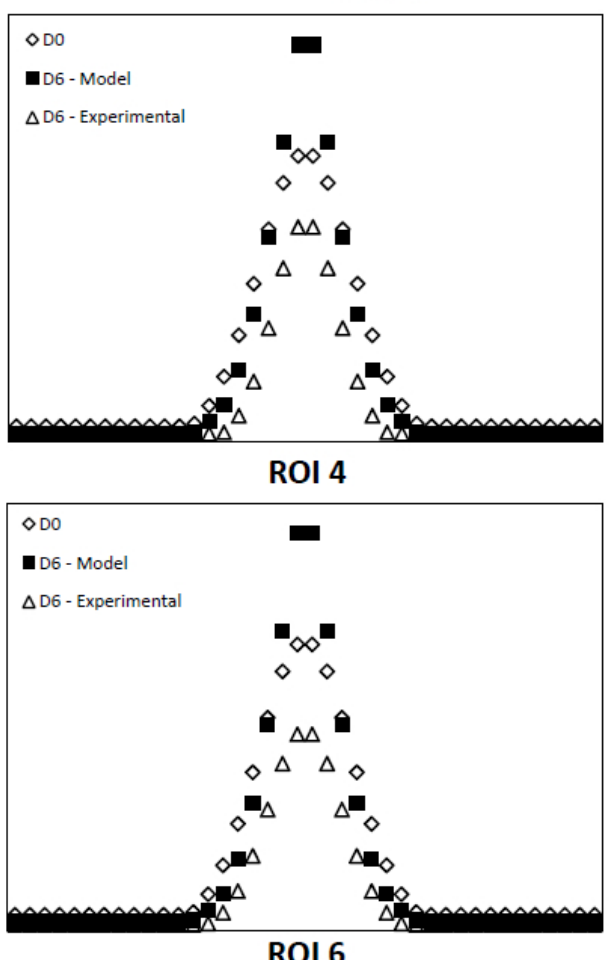

ROI 6

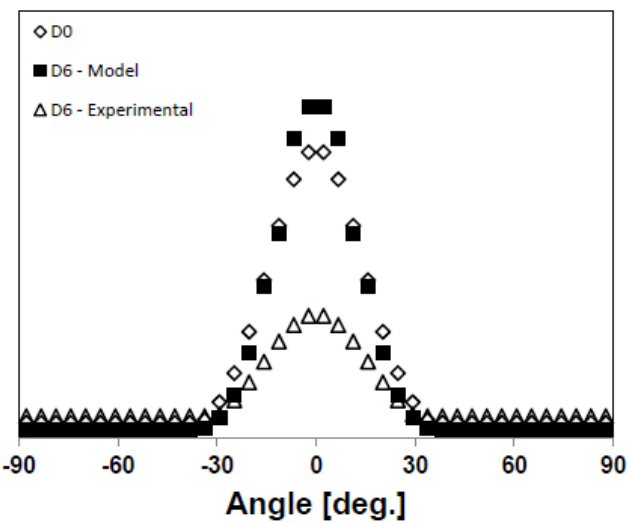

Figure 6.4: Predicted COL distribution after 6 days of simulated growth. The remodeling law did a poor job matching the experimental data. After this simulation, the maximum fiber strain data was inspected and it was determined that the proposed growth law would not be capable of predicting the observed changes in the COL fiber network. 

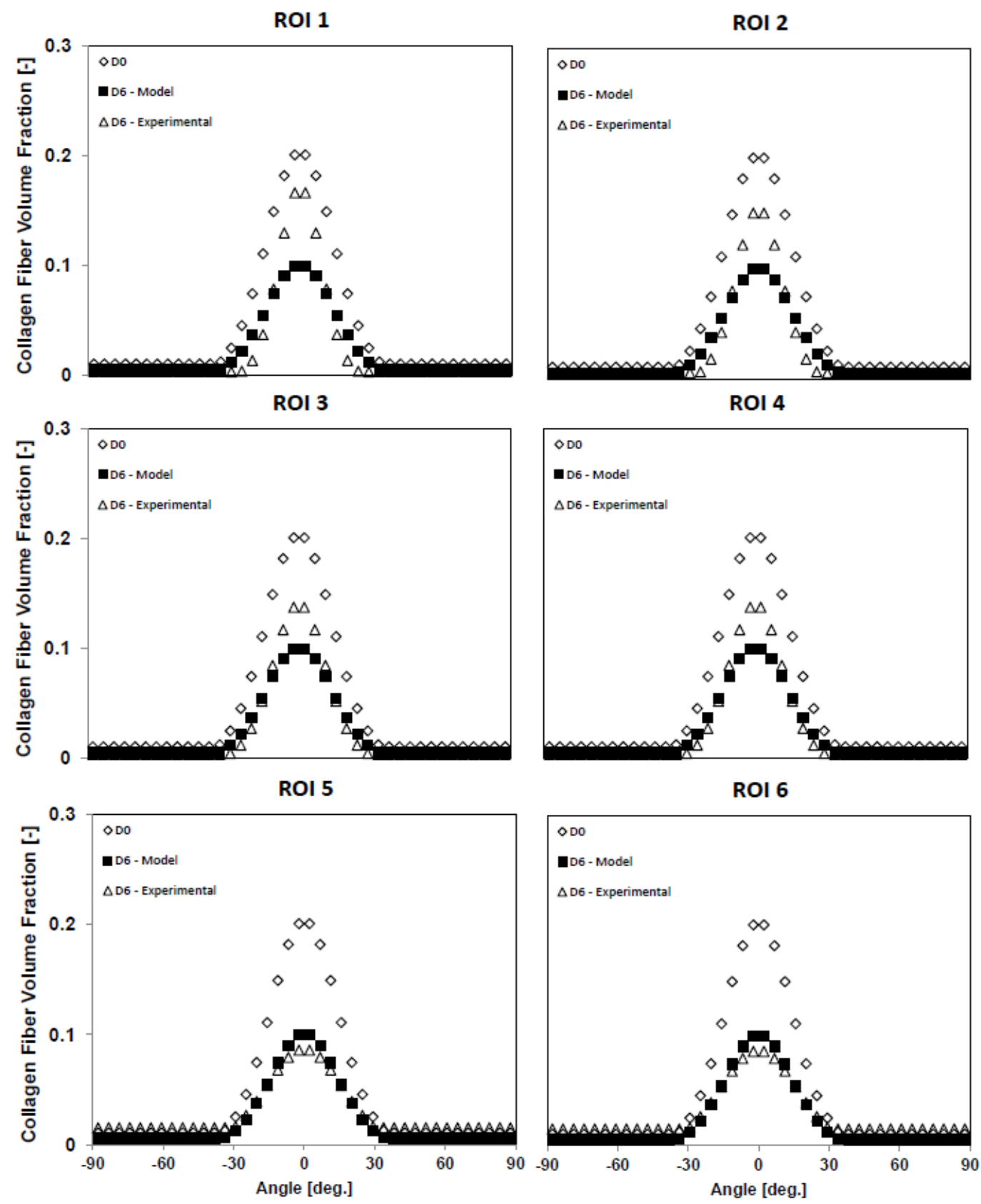

Figure 6.5: Predicted changes in COL fiber network with only the algorithm to predict remodeling due to chemical stimuli active. Note that the remodeling algorithm's predictions fit the peripheral regions of interest ( 5 and 6 ) better than the inner ones ( 1 and 2 ). This suggests that remodeling in the the peripheral region was an effect of the medium supplemented with IGF-1, not the dynamic unconfined compression. 
Table 6.2: Table of the final COL remodeling law parameters. The parameters for remodeling due to mechanical stimuli $\left(\eta\right.$ and $\left.E_{N, r}\right)$ were omitted because it was determined that the proposed algorithm for COL remodeling due to mechanical stimuli was not capable of predicting the experimental results.

\begin{tabular}{|c|c|c|}
\hline$\Upsilon$ & $\eta$ & $E_{N, r}$ \\
\hline$-0.11 \frac{1}{d a y}$ & - & - \\
\hline
\end{tabular}

For convenience, the COL remodling law is listed below.

$$
\frac{d \phi_{N}^{f}}{d t}=\Upsilon \phi_{N}^{f}+\kappa\left(E_{N}\right) \times \phi_{N}^{f}
$$

where $\times$ indicates scalar multiplication and

$$
\kappa\left(E_{N}\right)= \begin{cases}\eta & \text { for } E_{N, \max }>E_{N, g} \\ 0 & \text { for } E_{N, \max } \leq E_{N, g}\end{cases}
$$




\section{Chapter 7}

\section{Discussion}

Heterogeneous changes in GAG density and COL fiber network alignment were observed after culture with dynamic unconfined compression. The GAG density in the peripheral region of the sample was approximately $225 \%$ higher than that of the inner region. The COL network in the samples that received dynamic unconfined compression trended towards being more highly aligned in the inner region than the outer region, as demonstrated by a lower variance in average fiber angles, in the two innermost regions of interest. No significant difference between the variances in the average fiber angle were detected.

A poroelastic finite element model with an anisotropic COL fiber distribution and a GAG and COL remodeling subroutine was developed. The GAG remodeling algorithm related the change in GAG density linearly to the magnitude of the relative interstitial fluid velocity to change once the magnitude of the relative interstitial fluid velocity becomes greater than a threshold value. This algorithm was capable of predicting the changes in GAG density measured in the experimental portion of this study within 5\%. The threshold relative interstitial fluid velocity magnitude was found to be $0.60 \mu \mathrm{m} / \mathrm{s}$. 
The GAG density in the inner region of the D6 dynamic unconfined compression samples was lower than that of the D6 free-swelling controls. It is difficult to compare the change in COL mass between the inner region of the D6 dynamic unconfined compression samples and the D6 free-swelling controls because the initial wet weight of the inner region of the D6 samples is not available. The remodeling algorithm, which did not allow for GAG resorption, was able to predict the GAG density of the inner region of the dynamically loaded samples, which may suggest that the difference in measured densities was due to changes in tissue volume. Changes in tissue volume can be due to many factors, including changes in the COL fiber network.

It is possible that due to the dynamic unconfined compression, some GAG broke free and migrated towards the peripheral region of the disk. Generally, GAG is considered to be immobile in the extracellular matrix 66 67]. However, it has been shown that cyclic compression on cartilage/bone explants can lead to an increased loss of GAG and extracellular matrix to the media [68]. The displacement rates were lower in this study $(100 \mu \mathrm{m} / \mathrm{s}$ vs. 250 and $400 \mu \mathrm{m} / \mathrm{s}$ in the previous study). Future studies could analyze the media to determine if GAG is being expelled from the explant due to the dynamic unconfined compression.

The threshold value of relative interstitial fluid velocity magnitude for an increase in GAG density $v_{r}^{f / s}$ was found to be $0.60 \mu \mathrm{m} / \mathrm{s}$. A previous study found the threshold value for an increase in biosynthesis in $\mathrm{AC}$ from the patellofemoral groove of $1-2$ week old calves to be $0.25 \mu \mathrm{m} / \mathrm{s}$ [20]. The previous study used an isotropic biphasic model to predict the relative interstitial fluid velocity during dynamic unconfined compression. The discrepancy in remodeling threshold values may be due to the the assumption of isotropy. The model 
in this implemented a highly anisotropic distribution of COL fibers primarily oriented in the radial direction. This increased radial stiffness would result in a higher pressure gradient, likely resulting in higher relative interstitial fluid velocity magnitudes. Additionally, this study determined $v_{r}^{f / s}$ by correlating the magnitude of the relative interstitial fluid velocity with the location where the GAG remodeled, not where biosynthesis was upregulated.

The isotropic background distribution was added to account for the COL fibers in triangle elements that were measured by the qPLM data, but became numerically zero when the histogram of average fiber angles was fit with a normal distribution. An average fiber direction was assigned to each pixel. The pixels are $1.6 \mu \mathrm{m}$ by $1.6 \mu$ and COL fibers have a diameter of approximately $100 \mathrm{~nm}$ [69], so multiple fibers may be captured in each pixel. If multiple fibers are present in a pixel, the average fiber orientation is estimated from the birefringent response of all of the fibers and therefore contributions of fibers in in directions other than the average may not be accounted for. The background isotropic distribution estimates the contribution of these fibers.

A limitation of this study is that the day zero COL fiber distribution was estimated from qPLM data from newborn AC explants from the same source that had been cultured for four days in medium supplemented with $20 \%$ fetal bovine serum because no other data was available. It was assumed that the orientation of fibers did not change during unloaded culture. Due to the highly anisotropic distribution of fibers, it is unlikely that the fibers became more highly aligned. Experimental data from [27 supports this assumption.

An additional limitation of this study is the omission of a viscoelastic solid matrix. AC demonstrates flow-independent viscoelastic properties [70]. Poroviscolastic models of cartilage, which feature a viscoelastic solid phase, have 
been used to successfully model the mechanical response of articular cartilage in a variety of loading conditions, including unconfined compression, confined compression, and indentation 71, 72. Additionally, a poroviscoelastic model has successfully modeled the tension-compression asymmetry of AC by simultaneously predicting the response in uniaxial tension and compression [73. Anisotropic and bimodular biphasic models have had success in predicting the response of AC during unconfined compression [11,74 because fluid-flow dependent viscoelasticity is the dominant factor in the compressive behavior of AC $[75,76]$. Therefore, since this study focuses on unconfined compression, it was appropriate to omit intrinsic solid matrix viscoelasticity. Intrinsic solid matrix viscoelasticity should be added in future studies if simultaneously simulating multiple loading conditions, such as unconfined compression and tension, is desirable.

Future studies could further investigate the mechanisms of COL network realignment. The qPLM data from the treated samples suggested that the COL network had become more aligned in the radial direction, while maintaining the distribution in the peripheral region. The proposed growth laws were unable to predict this behavior, so others should be investigated. Other studies have successfully modeled collagen fiber reorientation in collagen gels using a growth law based on the Laplacian of Cauchy stress [36]. A law based on the gradient or Laplacian of fiber stress or strain may be appropriate. However, implementing such a law would require updating the user subroutines because state variables from adjacent integration points would have to be read in order to evaluate the derivatives and Abaqus does not support that natively. 
Further studies should be performed to elucidate the relationship between relative interstitial fluid velocity magnitude and the growth and remodeling of the GAG constituent. Techniques have been developed to measure GAG density with higher spatial resolution using contrast enhanced computed tomography [77,78]. Higher spatial resolution GAG density data would facilitate further exploration of the relationship between relative interstitial fluid velocity magnitude and GAG remodeling. 


\section{Bibliography}

[1] G. M. Williams, S. M. Klisch, and R. L. Sah, "Bioengineering cartilage growth, maturation, and form," Pediatric research, vol. 63, pp. 527-534, May 2008.

[2] J. A. Buckwalter and H. J. Mankin, "Articular cartilage repair and transplantation," Arthritis 83 Rheumatism, vol. 41, pp. 1331-1342, Aug. 1998.

[3] Centers for Disease Control and Prevention (CDC), "National and state medical expenditures and lost earnings attributable to arthritis and other rheumatic conditions-United States, 2003.," MMWR. Morbidity and mortality weekly report, vol. 56, pp. 4-7, Jan. 2007.

[4] G. J. V. M. G. van Osch, M. M. Brittberg, J. E. J. Dennis, Y. M. Y. Bastiaansen-Jenniskens, R. G. R. Erben, Y. T. Y. Konttinen, and F. P. F. Luyten, "Cartilage repair: past and future-lessons for regenerative medicine.," Audio, Transactions of the IRE Professional Group on, vol. 13, pp. 792-810, May 2009.

[5] A. G. A. McNickle, M. T. M. Provencher, and B. J. B. Cole, "Overview of existing cartilage repair technology.," Audio, Transactions of the IRE Professional Group on, vol. 16, pp. 196-201, Dec. 2008. 
[6] M. Hochberg, R. Altman, K. Brandt, B. Clark, P. Dieppe, M. Griffin, R. Moskowitz, and T. Schnitzer, "Guidelines for the medical management of osteoarthritis. Part I. Osteoarthritis of the hip. American College of Rheumatology.," Nov. 1995.

[7] W. L. W. Healy, R. R. Iorio, J. J. Ko, D. D. Appleby, and D. W. D. Lemos, "Impact of cost reduction programs on short-term patient outcome and hospital cost of total knee arthroplasty.," Journal of Bone and Joint Surgery (American), vol. 84-A, pp. 348-353, Mar. 2002.

[8] G. M. Williams, E. F. Chan, M. M. Temple-Wong, W. C. Bae, K. Masuda, W. D. Bugbee, and R. L. Sah, "Shape, loading, and motion in the bioengineering design, fabrication, and testing of personalized synovial joints," Journal of Biomechanics, vol. 43, pp. 156-165, Jan. 2010.

[9] J. S. J. Temenoff and A. G. A. Mikos, "Review: tissue engineering for regeneration of articular cartilage.," Biomaterials, vol. 21, pp. 431-440, Mar. 2000.

[10] G. A. Ateshian, M. A. Soltz, R. Mauck, I. Basalo, C. Hung, and W. Michael Lai, "The role of osmotic pressure and tension-compression nonlinearity in the frictional response of articular cartilage," Transport in porous media, vol. 50, no. 1, pp. 5-33, 2003.

[11] M. A. Soltz and G. A. Ateshian, "A Conewise Linear Elasticity mixture model for the analysis of tension-compression nonlinearity in articular cartilage.," Journal of Biomechanical Engineering, Transactions of the ASME, vol. 122, pp. 576-586, Dec. 2000. 
[12] C. Y. Huang, A. Stankiewicz, G. A. Ateshian, and V. C. Mow, "Anisotropy, inhomogeneity, and tension-compression nonlinearity of human glenohumeral cartilage in finite deformation," Journal of Biomechanics, vol. 38, no. 4, pp. 799-809, 2005.

[13] G. M. Williams, K. J. Dills, C. R. Flores, M. E. Stender, K. M. Stewart, L. M. Nelson, A. C. Chen, K. Masuda, S. J. Hazelwood, S. M. Klisch, and R. L. Sah, "Differential regulation of immature articular cartilage compressive moduli and Poisson's ratios by in vitro stimulation with IGF1 and TGF-beta1.," Journal of Biomechanics, vol. 43, pp. 2501-2507, Sept. 2010.

[14] N. T. Balcom, B. Berg-Johansen, K. J. Dills, J. R. Van Donk, G. M. Williams, A. C. Chen, S. J. Hazelwood, R. L. Sah, and S. M. Klisch, "In vitro Articular Cartilage Growth with Sequential Application of IGF1 and TGF-1 Enhances Volumetric Growth and Maintains Compressive Properties," Journal of biomechanical engineering, vol. 134, no. 3, p. 031001, 2012.

[15] T. P. Ficklin, G. Thomas, J. C. Barthel, A. Asanbaeva, E. J.-M. A. Thonar, K. Masuda, A. C. Chen, R. L. Sah, A. Davol, and S. M. Klisch, "Articular cartilage mechanical and biochemical property relations before and after in vitro growth.," Journal of Biomechanics, vol. 40, no. 16, pp. 3607-3614, 2007.

[16] A. K. Williamson, A. C. Chen, K. Masuda, E. J.-M. A. Thonar, and R. L. Sah, "Tensile mechanical properties of bovine articular cartilage: Variations with growth and relationships to collagen network components," Journal of Orthopaedic Research, vol. 21, no. 5, pp. 872-880, 2006. 
[17] A. Asanbaeva, K. Masuda, E. J.-M. A. Thonar, S. M. Klisch, and R. L. Sah, "Cartilage growth and remodeling: modulation of balance between proteoglycan and collagen network in vitro with beta-aminopropionitrile," Osteoarthritis and cartilage / OARS, Osteoarthritis Research Society, vol. 16, no. 1, pp. 1-11, 2008.

[18] A. Asanbaeva, K. Masuda, E. J.-M. A. Thonar, S. M. Klisch, and R. L. Sah, "Regulation of immature cartilage growth by IGF-I, TGF-beta1, BMP-7, and PDGF-AB: role of metabolic balance between fixed charge and collagen network," Biomechanics and Modeling in Mechanobiology, vol. 7, pp. 263-276, Aug. 2007.

[19] A. Asanbaeva, K. Masuda, E. J.-M. A. Thonar, S. M. Klisch, and R. L. Sah, "Mechanisms of cartilage growth: Modulation of balance between proteoglycan and collagen in vitro using chondroitinase ABC," Arthritis E Rheumatism, vol. 56, no. 1, pp. 188-198, 2006.

[20] M. D. Buschmann, Y.-J. Kim, M. Wong, E. H. Frank, E. B. Hunziker, and A. J. Grodzinsky, "Stimulation of aggrecan synthesis in cartilage explants by cyclic loading is localized to regions of high interstitial fluid flow," Archives of biochemistry and biophysics, vol. 366, pp. 1-7, June 1999.

[21] R. L. Sah, Y.-J. Kim, J.-Y. Doong, A. J. Grodzinsky, A. H. K. Plaas, and J. D. Sandy, "Biosynthetic response of cartilage explants to dynamic compression," Journal of orthopaedic research : official publication of the Orthopaedic Research Society, vol. 7, no. 5, pp. 619-636, 1989.

[22] A. C. Chen and R. L. Sah, "Effect of static compression on proteoglycan biosynthesis by chondrocytes transplanted to articular cartilage in vitro.," 
Journal of orthopaedic research : official publication of the Orthopaedic Research Society, vol. 16, pp. 542-550, Sept. 1998.

[23] R. L. Sah, J.-Y. Doong, A. J. Grodzinsky, A. H. K. Plaas, and J. D. Sandy, "Effects of compression on the loss of newly synthesized proteoglycans and proteins from cartilage explants," Archives of biochemistry and biophysics, vol. 286, pp. 20-29, Apr. 1991.

[24] G. E. Nugent, N. M. Aneloski, T. A. Schmidt, B. L. Schumacher, M. S. Voegtline, and R. L. Sah, "Dynamic shear stimulation of bovine cartilage biosynthesis of proteoglycan 4," Arthritis \& Rheumatism, vol. 54, no. 6, pp. 1888-1896, 2006.

[25] K. W. Li, A. K. Williamson, A. S. Wang, and R. L. Sah, "Growth responses of cartilage to static and dynamic compression," Clinical orthopaedics and related research, pp. S34-48, Oct. 2001.

[26] T. P. Ficklin, A. Davol, and S. M. Klisch, "Simulating the growth of articular cartilage explants in a permeation bioreactor to aid in experimental protocol design," Journal of biomechanical engineering, vol. 131, p. 041008, Apr. 2009.

[27] M. Stender, C. Raub, R. Shirazi, K. Yamauchi, V. Pasquale, R. L. Sah, S. J. Hazelwood, and S. M. Klisch, "Effects of TGF-b1 and IGF-1 on Articular Cartilage Collagen Fiber Modulus: Prediction Using a Continuous Fiber Distribution Model and qPLM.," In Preparation.

[28] V. C. Mow, S. C. Kuei, W. M. Lai, and C. G. Armstrong, "Biphasic creep and stress relaxation of articular cartilage in compression? Theory and 
experiments," Journal of biomechanical engineering, vol. 102, pp. 73-84, Feb. 1980.

[29] P. S. Donzelli, R. L. Spilker, G. A. Ateshian, and V. C. Mow, "Contact analysis of biphasic transversely isotropic cartilage layers and correlations with tissue failure.," Journal of Biomechanics, vol. 32, pp. 1037-1047, Oct. 1999.

[30] R. Krishnan, S. Park, F. Eckstein, and G. A. Ateshian, "Inhomogeneous cartilage properties enhance superficial interstitial fluid support and frictional properties, but do not provide a homogeneous state of stress," Journal of biomechanical engineering, vol. 125, p. 569, 2003.

[31] G. A. Ateshian, W. H. Warden, J. J. Kim, R. P. Grelsamer, and V. C. Mow, "Finite deformation biphasic material properties of bovine articular cartilage from confined compression experiments.," Journal of Biomechanics, vol. 30, pp. 1157-1164, Nov. 1997.

[32] S. M. Klisch, "A bimodular polyconvex anisotropic strain energy function for articular cartilage," Journal of biomechanical engineering, vol. 129, pp. 250-258, Apr. 2007.

[33] R. L. Spilker, P. S. Donzelli, and V. C. Mow, "A transversely isotropic biphasic finite element model of the meniscus," Journal of Biomechanics, vol. 25, no. 9, pp. 1027-1045, 1992.

[34] A. J. Grodzinsky, M. E. Levenston, M. Jin, and E. H. Frank, "Cartilage tissue remodeling in response to mechanical forces.," Annual review of biomedical engineering, vol. 2, pp. 691-713, 2000. 
[35] V. C. Mow and W. C. Hayes, Basic orthopaedic biomechanics. Lippincott Williams \& Wilkins, 1997.

[36] M. Kroon, "A continuum mechanics framework and a constitutive model for remodelling of collagen gels and collagenous tissues," Journal of the Mechanics and Physics of Solids, vol. 58, pp. 918-933, Apr. 2010.

[37] R. E. CRAine, A. E. GREEN, and P. M. NAGHDI, "A MiXturE OF VISCOUS ELASTIC MATERIALS WITH DIFFERENT CONSTITUENT TEMPERATURES," The Quarterly Journal of Mechanics and Applied Mathematics, vol. 23, no. 2, pp. 171-184, 1970.

[38] A. E. GREEN and P. M. NAGHDI, "On Basic Equations For Mixtures," The Quarterly Journal of Mechanics and Applied Mathematics, vol. 22, no. 4, pp. 427-438, 1969.

[39] A. E. GREEN and P. M. NAGHDI, "A note on mixtures," International Journal of Engineering Science, vol. 6, no. 11, pp. 631-635, 1968.

[40] R. J. ATKIN and R. E. CRAINE, "CONTINUUM THEORIES OF MIXTURES: BASIC THEORY AND HISTORICAL DEVELOPMENT," The Quarterly Journal of Mechanics and Applied Mathematics, vol. 29, no. 2, pp. 209-244, 1976.

[41] M. H. Holmes and V. C. Mow, "The nonlinear characteristics of soft gels and hydrated connective tissues in ultrafiltration.," Journal of Biomechanics, vol. 23, no. 11, pp. 1145-1156, 1990.

[42] S. Klisch and J. Lotz, "A special theory of biphasic mixtures and experimental results for human annulus fibrosus tested in confined compres- 
sion.," Journal of Biomechanical Engineering, Transactions of the ASME, vol. 122, pp. 180-188, Apr. 2000.

[43] M. Stender, Predicting Articular Cartilage Constituent Material Properties Following In Vitro Growth Using A Proteoglycan-Collagen Mixture Model. PhD thesis, California Polytechnic State University - San Luis Obispo, Mar. 2011.

[44] A. Williamson, A. Chen, and R. L. Sah, "Compressive properties and function - composition relationships of developing bovine articular cartilage," Journal of Orthopaedic Research, vol. 19, no. 6, pp. 1113-1121, 2001.

[45] A. I. Maroudas, "Balance between swelling pressure and collagen tension in normal and degenerate cartilage.," Nature, vol. 260, pp. 808-809, Apr. 1976.

[46] Y. Kim, L. Bonassar, and A. J. Grodzinsky, "The role of cartilage streaming potential, fluid flow and pressure in the stimulation of chondrocyte biosynthesis during dynamic compression," Journal of Biomechanics, vol. 28, no. 9, pp. 1055-1066, 1995.

[47] T. Davisson, R. L. Sah, and A. Ratcliffe, "Perfusion increases cell content and matrix synthesis in chondrocyte three-dimensional cultures.," Tissue engineering, vol. 8, pp. 807-816, Oct. 2002.

[48] D. D. Pazzano, K. A. K. Mercier, J. M. J. Moran, S. S. S. Fong, D. D. D. DiBiasio, J. X. J. Rulfs, S. S. S. Kohles, and L. J. L. Bonassar, "Comparison of chondrogensis in static and perfused bioreactor culture.," Biotechnology Progress, vol. 16, pp. 893-896, Jan. 2000. 
[49] P. Julkunen, J. Iivarinen, P. A. Brama, J. Arokoski, J. S. Jurvelin, and H. J. Helminen, "Maturation of collagen fibril network structure in tibial and femoral cartilage of rabbits.," Osteoarthritis and cartilage / OARS, Osteoarthritis Research Society, vol. 18, pp. 406-415, Mar. 2010.

[50] M. C. van Turnhout, H. Schipper, B. Engel, W. Buist, S. Kranenbarg, and J. L. van Leeuwen, "Postnatal development of collagen structure in ovine articular cartilage.," BMC developmental biology, vol. 10, p. 62, 2010.

[51] J. Rieppo, M. M. Hyttinen, E. Halmesmaki, H. Ruotsalainen, A. Vasara, I. Kiviranta, J. S. Jurvelin, and H. J. Helminen, "Changes in spatial collagen content and collagen network architecture in porcine articular cartilage during growth and maturation.," Osteoarthritis and cartilage / OARS, Osteoarthritis Research Society, vol. 17, pp. 448-455, Apr. 2009.

[52] M. M. Hyttinen, J. Holopainen, P. R. van Weeren, E. C. Firth, H. J. Helminen, and P. A. J. Brama, "Changes in collagen fibril network organization and proteoglycan distribution in equine articular cartilage during maturation and growth.," Journal of anatomy, vol. 215, pp. 584-591, Nov. 2009.

[53] M. M. Hyttinen, J. P. Arokoski, J. J. Parkkinen, M. J. Lammi, T. Lapveteläinen, K. Mauranen, K. Király, M. I. Tammi, and H. J. Helminen, "Age matters: collagen birefringence of superficial articular cartilage is increased in young guinea-pigs but decreased in older animals after identical physiological type of joint loading.," Osteoarthritis and cartilage / OARS, Osteoarthritis Research Society, vol. 9, pp. 694-701, Nov. 2001. 
[54] N. J. B. Driessen, W. Wilson, C. V. C. Bouten, and F. P. T. Baaijens, "A computational model for collagen fibre remodelling in the arterial wall," Journal of theoretical biology, vol. 226, pp. 53-64, Jan. 2004.

[55] E. B. Hunziker, E. Kapfinger, and J. Geiss, "The structural architecture of adult mammalian articular cartilage evolves by a synchronized process of tissue resorption and neoformation during postnatal development.," Osteoarthritis and cartilage, vol. 15, pp. 403-413, Apr. 2007.

[56] J. J. Rieppo, J. J. Hallikainen, J. S. J. Jurvelin, I. I. Kiviranta, H. J. H. Helminen, and M. M. M. Hyttinen, "Practical considerations in the use of polarized light microscopy in the analysis of the collagen network in articular cartilage.," Microscopy Research and Technique, vol. 71, pp. 279287, Apr. 2008.

[57] R. Shirazi, V. Pasquale, R. L. Sah, and S. M. Klisch, "Modeling collagen fibrils using a continuous volume fraction distribution function for use in nonlinear anisotropic polyconvex mixture models of articular cartilage," Mathematics and Mechanics of Solids.

[58] M. D. Buschmann and A. J. Grodzinsky, "A molecular model of proteoglycan-associated electrostatic forces in cartilage mechanics.," Journal of Biomechanical Engineering, Transactions of the ASME, vol. 117, pp. 179-192, May 1995.

[59] F. Lei and A. Szeri, "The influence of fibril organization on the mechanical behaviour of articular cartilage," Proceedings of the Royal Society A: Mathematical, Physical and Engineering Science, vol. 462, no. 2075, pp. 3301-3322, 2006. 
[60] R. W. Farndale, D. J. Buttle, and A. J. Barrett, "Improved quantitation and discrimination of sulphated glycosaminoglycans by use of dimethylmethylene blue," Biochimica et Biophysica Acta (BBA) - General Subjects, vol. 883, no. 2, pp. 173-177, 1986.

[61] J. F. Woessner Jr, "The determination of hydroxyproline in tissue and protein samples containing small proportions of this imino acid," Archives of biochemistry and biophysics, vol. 93, no. 2, pp. 440-447, 1961.

[62] P. J. Basser, R. Schneiderman, R. A. Bank, E. Wachtel, and A. Maroudas, "Mechanical Properties of the Collagen Network in Human Articular Cartilage as Measured by Osmotic Stress Technique," Archives of biochemistry and biophysics, vol. 351, pp. 207-219, Mar. 1998.

[63] K. K. Kam, "Poroelastic Finite Element Analysis of a Heterogeneous Articular Cartilage Explant Under Dynamic Compression in ABAQUS," Master's thesis, California Polytechnic State University - San Luis Obispo, San Luis Obispo, June 2011.

[64] S. M. Klisch, A. Asanbaeva, S. R. Oungoulian, K. Masuda, E. J.-M. A. Thonar, A. Davol, and R. L. Sah, "A cartilage growth mixture model with collagen remodeling: validation protocols," Journal of biomechanical engineering, vol. 130, p. 031006, June 2008.

[65] D. Systems, ABAQUS 6.7 Analysis User's Manual.

[66] C. R. Roberts and P. D. Paré, "Composition changes in human tracheal cartilage in growth and aging, including changes in proteoglycan structure.," The American journal of physiology, vol. 261, pp. L92-101, Aug. 1991. 
[67] T. E. Hardingham, "The role of link-protein in the structure of cartilage proteoglycan aggregates.," The Biochemical journal, vol. 177, pp. 237247, Jan. 1979.

[68] M. Thibault, A. R. Poole, and M. D. Buschmann, "Cyclic compression of cartilage/bone explants in vitro leads to physical weakening, mechanical breakdown of collagen and release of matrix fragments.," Journal of orthopaedic research: official publication of the Orthopaedic Research Society, vol. 20, pp. 1265-1273, Nov. 2002.

[69] A. A. Changoor, M. M. Nelea, S. S. Méthot, N. N. Tran-Khanh, A. A. Chevrier, A. A. Restrepo, M. S. M. Shive, C. D. C. Hoemann, and M. D. M. Buschmann, "Structural characteristics of the collagen network in human normal, degraded and repair articular cartilages observed in polarized light and scanning electron microscopies.," Audio, Transactions of the IRE Professional Group on, vol. 19, pp. 1458-1468, Dec. 2011.

[70] W. C. Hayes and A. J. Bodine, "Flow-independent viscoelastic properties of articular cartilage matrix," Journal of Biomechanics, vol. 11, no. 8-9, pp. 407-419, 1978.

[71] W. Wilson, C. C. van Donkelaar, B. van Rietbergen, K. Ito, and R. Huiskes, "Stresses in the local collagen network of articular cartilage: a poroviscoelastic fibril-reinforced finite element study," Journal of Biomechanics, vol. 37, pp. 357-366, Mar. 2004.

[72] W. Wilson, C. C. van Donkelaar, B. van Rietbergen, and R. Huiskes, "A fibril-reinforced poroviscoelastic swelling model for articular cartilage," Journal of Biomechanics, vol. 38, pp. 1195-1204, June 2005. 
[73] C. Y. Huang, V. C. Mow, and G. A. Ateshian, "The role of flowindependent viscoelasticity in the biphasic tensile and compressive responses of articular cartilage.," Journal of Biomechanical Engineering, Transactions of the ASME, vol. 123, pp. 410-417, Oct. 2001.

[74] B. Cohen, W. M. Lai, and V. C. Mow, "A transversely isotropic biphasic model for unconfined compression of growth plate and chondroepiphysis.," Journal of Biomechanical Engineering, Transactions of the ASME, vol. 120, pp. 491-496, Aug. 1998.

[75] S. S. Park and G. A. G. Ateshian, "Dynamic response of immature bovine articular cartilage in tension and compression, and nonlinear viscoelastic modeling of the tensile response.," Journal of Biomechanical Engineering, Transactions of the ASME, vol. 128, pp. 623-630, Aug. 2006.

[76] L. P. L. Li, W. W. Herzog, R. K. R. Korhonen, and J. S. J. Jurvelin, "The role of viscoelasticity of collagen fibers in articular cartilage: axial tension versus compression.," Medical Engineering \&3 Physics, vol. 27, pp. 51-57, Jan. 2005.

[77] A. W. Palmer, R. E. Guldberg, and M. E. Levenston, "Analysis of cartilage matrix fixed charge density and three-dimensional morphology via contrast-enhanced microcomputed tomography.," Proceedings of the $\mathrm{Na}$ tional Academy of Sciences of the United States of America, vol. 103, pp. 19255-19260, Dec. 2006.

[78] P. N. Bansal, N. S. Joshi, V. Entezari, B. C. Malone, R. C. Stewart, B. D. Snyder, and M. W. Grinstaff, "Cationic contrast agents improve quantification of glycosaminoglycan (GAG) content by contrast enhanced CT 
imaging of cartilage," Journal of orthopaedic research : official publication of the Orthopaedic Research Society, vol. 29, pp. 704-709, May 2011. 